\title{
Dynamic Performance of Mobile Haptic Interfaces
}

\author{
Alessandro Formaglio, Associate Member, IEEE, Domenico Prattichizzo, Member, IEEE, Federico Barbagli, \\ and Antonio Giannitrapani, Member, IEEE
}

\begin{abstract}
The increasing demand for virtual reality applications in several scientific disciplines feeds new research perspectives dealing with robotics, automation, and computer science. In this context, one of the topics is the design of advanced force-feedback devices allowing not only kinesthetic interaction with virtual objects but also locomotion and navigation inside virtual worlds. This has the main advantage to stimulate human vestibular apparatus, thus increasing the overall realism of simulation. Particularly, this paper deals with mobile haptic interfaces (MHIs), built by combining standard force-feedback devices with mobile platforms. We investigated which factors may affect the transparency of this kind of devices, identifying in mobile robot dynamics a possible cause of loss of transparency. Hence, in this paper, we present a method to analyze dynamic performance of an $\mathrm{MHI}$ and some basic guidelines to design controller in order to meet desired specifications. Experimental validation of the theoretical results is reported.
\end{abstract}

Index Terms-Design, haptics, mobile robots, performance analysis.

\section{INTRODUCTION}

I N THE LAST decade, Virtual Reality (VR) applications have seen a great deal of development. In this context, a fundamental innovation was the introduction of haptic interfaces, since this allowed kinesthetic interaction between users and virtual environments (VEs), thus highly increasing the overall realism of VR applications. Since the first force-feedback prototype was introduced, haptic interfaces have been considerably improved. And yet, the performance of haptic interfaces is still limited (see [1] for an extensive description of performance metrics for haptic interfaces).

One of the most apparent limitations of most haptic interfaces to date is their limited workspace, which normally ranges between a few cubic centimeters typical of most desktop devices [2], [3] to a few cubic meters typical of most exoskeleton-type devices [4]. Typically, users are constrained to stand still within the workspace of the haptic device (HD) and interact in a limited volume depending on the device kinematics and dimensions.

The problem of extending the workspace of haptic interfaces has been addressed in the past. Four main approaches have been proposed: building larger devices, using locomotive interfaces, extending workspace by allowing users to navigate through VEs via software, and designing mobile haptic interfaces (MHIs). Examples of the first approach are, for instance, the scaleable

Manuscript received July 12, 2007; revised December 21, 2007. This paper was recommended for publication by Associate Editor H. R. Choi and Editor $\mathrm{K}$. Lynch upon evaluation of the reviewers' comments.

A. Formaglio, D. Prattichizzo, and A. Giannitrapani are with the Dipartimento di Ingegneria dell'Informazione, University of Siena, 53100 Siena, Italy (e-mail: alex@dii.unisi.it; prattichizzo@dii.unisi.it; giannitrapani@dii.unisi.it).

F. Barbagli is with the Stanford Robotics Laboratory, Stanford University, CA 94305-5404 USA (e-mail: fed_barbagli@hansenmedical.com).

Color versions of one or more of the figures in this paper are available online at http://ieeexplore.ieee.org.

Digital Object Identifier 10.1109/TRO.2008.924262

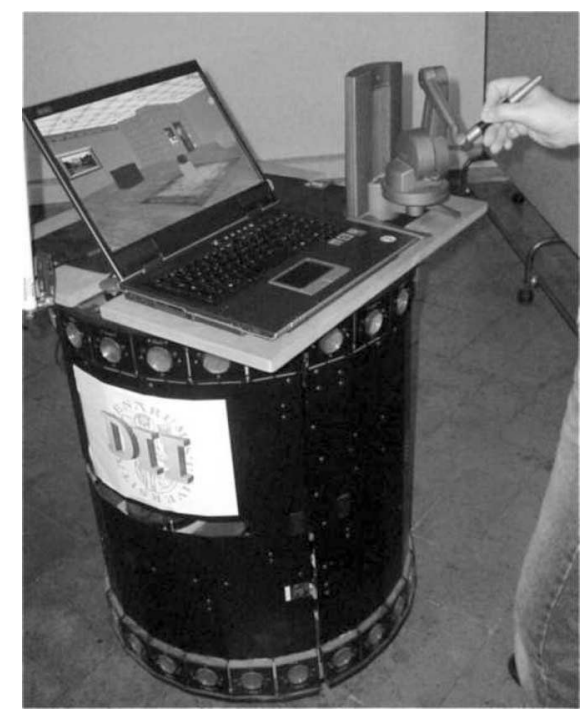

Fig. 1. MHI based on the holonomic Nomad mobile robot and a desktop PHANTOM.

spidar, a complex structure made up of a steel frame and a cabled end-effector that allows the user to haptically interact within a considerably large workspace (featuring a volume of about $27 \mathrm{~m}^{3}$ ) [5]. Examples of the second approach are, for instance, the hyper-redundant haptic interface proposed in [6], which features ten degrees of freedom (DoFs), allowing the user with some form of locomotion, consisting of rotations about the center of the workspace. Examples of the third approach are, for instance, the workspace drift control, i.e., a control strategy that transparently shifts the physical workspace of the device mapped inside the virtual world thus providing the illusion of haptic interaction with an unlimited workspace [7]. Examples of the fourth approach are, for instance, the cobots, i.e., devices that allow users to track a precomputed path by displaying virtual constraints (walls) in the real environment [8], and MHIs, i.e., interfaces obtained combining desktop HDs with mobile platforms (MPs) [9], [10].

In this paper, we will focus our attention on MHI (see Fig. 1), as they are the only class of interfaces allowing for true unlimited planar workspace. The main difference between MHI and grounded HDs is that the spatial workspace strongly depends on dynamic performance of the overall system and its unboundedness is guaranteed provided that some dynamic performance limitations are satisfied. This paper extends preliminary results presented in [10] and [11]. The goal of this paper is to analyze dynamic performance of MHIs and to provide the Haptics and Robotics communities with some useful guidelines on how to design MHI and their control algorithms in order to obtain a given performance specification 
and unbounded MHI workspace. The main problem facing an MHI designer is that typically the MP of MHIs features slower dynamics than the system composed of the HD and its user, and thus, it is likely that the MP will not be able to track any type of motion generated by the user. As a result, there will be user motions during which the HD end-effector reaches its physical workspace boundary. This situation corresponds to unwanted reaction forces on the operator's hand, which affect the overall level of realism. As such, MHIs can be seen as interfaces featuring unlimited spatial workspace but much more limited dynamic performance than standard desktop devices, and thus, present their designers with technical challenges usually not faced when employing more standard HDs.

The main contribution of this paper is the analysis of dynamic performance of an MHI and the computation of the relationships between system parameters and performance. Theoretical results are presented and validated experimentally on an MHI composed by a PHANToM premium haptic interface with a Nomad XR4000 mobile robot.

The MP model considered is simple to understand and easy to tune, but does not take into account all possible nonlinearities that are present in reality. Experimental results support this choice; however, we also report brief analysis addressed to evaluate how and to which extent nonlinearities we neglect are relevant with respect to the linear approach.

The paper is structured as follows. Section II introduces the dynamic model of an MHI. Section III shows the formalization of the problem of transparency for an MHI. Such a problem is studied in Section IV in terms of force-rendering performance, whereas in Section V, we present some basic metrics that allow users to characterize the motion performance of an MHI. Section VI illustrates the application of performance analysis in order to design an MHI controller. Section VII presents the extension of performance analysis to a more realistic scenario in which actuator saturation has been taken into account. In Section VIII, experiments that have been carried out to validate theoretical analysis are described. In Section IX, we provide a further generalization of some results in the frequency domain. Finally, in Section X, some conclusions are drawn.

\section{Modeling Mobile Haptic INTERfaces}

MHIs are designed to allow the interaction between user and objects displaced in large VEs [9]. For this purpose, MHIs are made up of two main components: an MP and an impedancetype HD, grounded to the MP (see Fig. 1). The latter provides kinesthetic interaction, while the former tracks the locomotion of the user in the virtual world. With this strategy, the MP moves the HD workspace, mapped inside the VE, toward the area of interest of the user, according to her/his real motion.

Despite the heterogeneity of the HDs and mobile robots that can be employed, all MHIs share some common features.

1) A single-contact-point MHI typically is characterized by kinematic redundancy, i.e., dimensions of the operational space are less than MHI DoFs. For example, an MHI realized combining the PHANToM HD (three DoFs) and the Nomad XR4000 MP (three DoFs) globally features six DoFs, while operational space has only three dimensions.

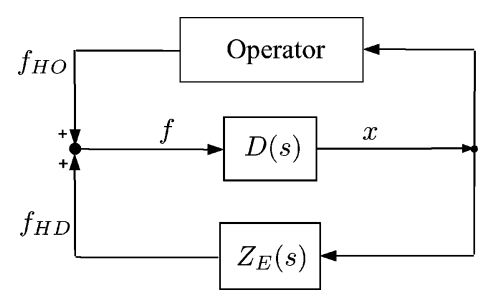

Fig. 2. Scheme of a system for haptic interaction between user and VE, using a desktop force-feedback device.

2) MPs are generally position (or velocity) controlled, while impedance HDs are force controlled. This leads to a hybrid position/force (or velocity/force) control scheme for an MHI.

3) While HDs are designed to feature high backdriveability and transparency, MPs are usually characterized by high mass and inertia. Therefore, an MHI simultaneously presents slow position- (or velocity-) controlled dynamics, and fast force-controlled dynamics.

The model adopted in this paper originates from the aforementioned observations, and derives from the one proposed by Nitzsche et al. in [9]. The basic idea is to make the MP track the motion of the operator, thus always driving the HD end-effector toward the center of its workspace. The advantage of this policy is twofold. First, it allows the HD to render forces in a configuration of maximum structural stiffness. Second, as long as the HD end-effector lies close to the center of its workspace during the simulation, it gives the larger margin for the tracking error due to sudden movements performed by user's hand. On the other hand, forces are rendered using standard constrainedbased methods such as the proxy algorithm [12].

This section describes the model of an MHI able to move and render forces along one direction. Refer to this direction as the $x$-axis.

First of all, let us consider the classic scheme that was introduced by Colgate in [13] (see Fig. 2). It models haptic interaction between human operator and a VE through a grounded HD: $f$ is the total force applied to the end-effector; $x$ represents the position of the end-effector; $D(s)$ models the end-effector reflected inertia felt by the operator; finally, $Z_{E}(s)$ is the transfer function of the VE impedance. Note that although this simplified model is usually adopted to represent interaction with virtual walls, it can be generalized to more complex cases by adding a collision-detection block.

The dynamic model of an MHI is derived from the model proposed for grounded HDs. Refer to Fig. 3 and let $\Sigma_{W}$ be the base reference frame attached to the world and let $\Sigma_{M}$ be the reference frame attached to the MP base. Note that reference frame $\Sigma_{M}$ is centered at a position where both the distance from the HD workspace and some manipulability indexes are optimized [14], [15]. In Fig. 3, $x_{H}$ represents the position of the HD end-effector with respect to $\Sigma_{W}, x_{M}$ is the position of frame $\Sigma_{M}$ with respect to $\Sigma_{W}$, and $e=x_{H}-x_{M}$ is the position of the HD end-effector with respect to $\Sigma_{M}$. The functional scheme of the MHI is reported in Fig. 4. The MP dynamics is modeled through the transfer function $H(s)$ having as input the reference 


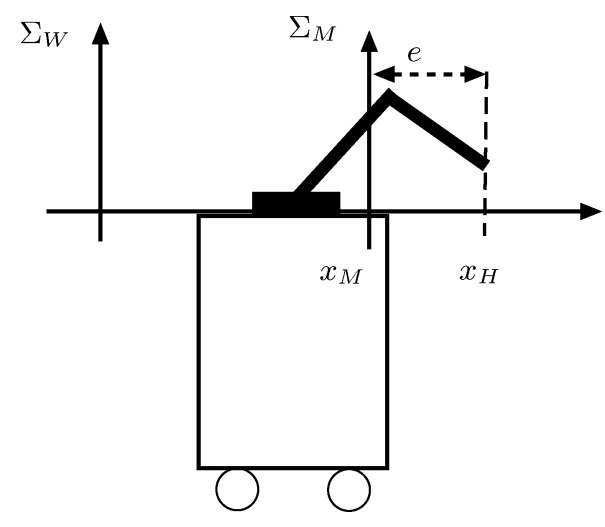

Fig. 3. Reference frames and position vectors of the MHI.

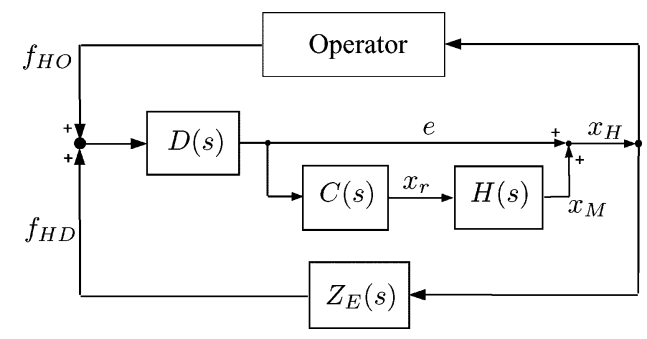

Fig. 4. Scheme of a system for haptic interaction between user and VE, using an MHI.

position $x_{r}$ commanded to the MP and as output its position $x_{M}$ in the world frame. The transfer function of the MP control system is $C(s)$ whose input $e$ is also referred to as tracking error. Note that being $e$ the position of the HD end-effector in frame $\Sigma_{M}$, it is directly available through HD sensor readings.

The MP controller $C(s)$ is chosen in order to track the endeffector position with respect to the world frame, i.e., to bring the tracking error $e$ to zero. In this paper, a proportional-derivative (PD) controller is considered

$$
C(s)=K_{p}+K_{d} s
$$

The impedance model of a virtual object is chosen as a springdamper system, yielding

$$
Z_{E}(s)=k_{E}+s b_{E}
$$

where $k_{E}$ and $b_{E}$ are virtual object stiffness and damping, respectively. A simple linear model is adopted for the MP. Trading off accuracy for simplicity, we decided to represent the MP as a damped mass subject to elastic forces; hence, the MP transfer function $H(s)$ takes on the form:

$$
H(s)=\frac{k_{M}}{s\left(m_{M} s+b_{M}\right)}
$$

where the mass $m_{M}$, the spring constant $k_{M}$, and the damping factor $b_{M}$ are the parameters characterizing the MP. The advantages of choosing such a simple model are that it is characterized by a small set of parameters that can be easily identified, its physical interpretation is straightforward, and, above all, it allows to compute analytic relationships between its parameters and the achievable motion performance of the MHI. On the other hand, such a model cannot account for nonlinear dynamics that are present in reality, e.g., actuator saturation and bounded accelerations.

\section{MHI TRANSPARENCY ANALYSIS}

Impedance-type HDs, such as the PHANToM and the Omega, should render impedances ranging from $Z_{\mathrm{min}}$, corresponding to movements in free space, to a maximum value $Z_{\max }$ that depends on a multitude of factors [13]. The interval $\left[Z_{\min }, Z_{\max }\right]$ is referred to as $Z$-width. HDs are designed in order to have low reflected inertia and friction, i.e., $Z_{\mathrm{min}} \approx 0$, thus preserving good haptic transparency while moving in free space.

In this section, we study features such as inertia, maximum forces, and $Z$-width for MHIs. The first remark is that mobile robots typically feature slower dynamics than the HD endeffector handled by the operator. Because of this, it is unlikely for the MP to be able to track any type of reference trajectory generated by the user. As a result of fast user motions, it is possible for the HD end-effector to reach its workspace boundary. This situation corresponds to a kinematic singularity of the HD manipulator that inhibits motion along some direction. This, in turn, corresponds to unwanted reaction forces on the operator's hand. This occurrence represents a remarkable loss of realism, since the user would experience forces not associated to virtual contact but due to hardware limitation.

The dynamic equilibrium between the operator and the MHI is represented by the following equation:

$$
f_{\mathrm{HO}}(s)=Z_{E}(s) x_{H}(s)+f_{D}(s)+f_{\mathrm{WS}}(s)
$$

where $f_{\mathrm{HO}}$ is exerted by the human operator; $Z_{E} x_{H}$ is the reaction force, computed using standard haptic rendering techniques [12] and resulting in a force proportional to the penetration of the HD end-effector inside a virtual object (see [13]); $f_{D}$ is the force due to MHI dynamics (inertia and friction), and finally, $f_{\mathrm{Ws}}$ represents possible spurious forces due to the HD end-effector workspace boundary.

The impedance $Z^{*}(s)$ really felt by the user is defined as

$$
Z^{*}(s)=\frac{f_{\mathrm{HO}}(s)}{x_{H}(s)} .
$$

The transparency requirement means that $Z^{*}(s) \approx Z_{E}(s)$, thus it stems from (3) that transparency is achieved if:
a) $f_{D} \approx 0 \quad$ (Low reflected inertia)
b) $f_{\mathrm{WS}}=0 . \quad$ (Within workspace boundaries)

The rest of the work focuses on identifying the conditions for which these two requirements are satisfied.

Requirement (4a) is typically satisfied for grounded HDs, whose mechanical design generally guarantees high backdriveability of the end-effector. Nevertheless, it must be studied whether inertia, maximum forces, and $Z$-width of an MHI are simply inherited from the employed HD or dynamics of MP significantly influences them.

As regard to specification (4b), the effects produced by a spurious force $f_{\mathrm{WS}} \neq 0$ cannot be neglected, but it is possible to derive sufficient conditions ensuring $f_{\mathrm{WS}}=0$. To that end, the 


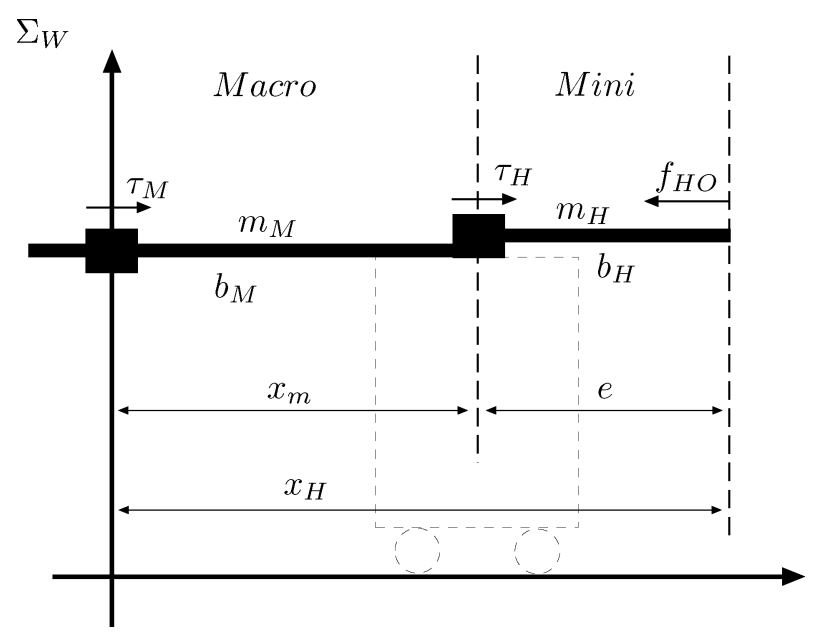

Fig. 5. Dynamical model consisting of a 2-DoF MHI featuring onedimensional operational space.

HD end-effector should never reach its workspace boundary, i.e., $|e(t)|$ must be bounded. Such a bound reflects on input signal $x_{H}$; hence, the target of the second part of our study consists of determining what conditions should satisfy the input $x_{H}$ in order for $f_{\mathrm{WS}}=0$ to hold.

\section{Force RENDERING PERFORMANCE}

In order to verify whether condition (4a) is satisfied for an MHI, inertial properties, $Z$-width, and maximum forces for an MHI have been examined. In the following analysis, we will consider the scenario in which the end-effector moves inside its workspace, i.e., throughout this section, we will assume that condition (4b) holds. The latter assumption will be removed in Section V, where sufficient conditions for (4b) to be true will be presented.

\section{A. Inertial Properties}

As pointed out in the previous section, the global reflected inertia perceived by the user at the end-effector should be ideally zero, but actually friction and inertia of mechanical systems affects end-effector dynamics. Before analyzing inertial properties for an MHI, we briefly recall that it is a kinematically redundant system, hence, as shown in [16], it can be decomposed into two subsystems referred to as mini and macro structures: the mini structure is defined as the smallest distal set of DoFs that can completely span the operational space; the macro structure connects the mini one to the ground. In [16], the author proved that the inertial properties of the overall redundant system are bounded above by the inertial properties of the mini structure alone.

Such a result applies also to an MHI. Let us consider a 2-DoF MHI featuring one-dimensional operational space (see Fig. 5), where $x_{H}$ represents the end-effector position with respect to the global reference frame $\Sigma_{W}$, while $x_{m}$ and $e$ represent the joint space coordinates; $m_{M}$ and $m_{H}$ are MP and HD masses, respectively; $b_{M}$ and $b_{H}$ are the two subsystems damping coefficients; $\tau_{M}$ and $\tau_{H}$ are the forces exerted by MP and HD, respectively; $f_{\mathrm{HO}}$ is the force exerted by the human operator. The mini/macro decomposition can be performed as shown in Fig. 5. The mini structure is represented by the HD, which features one DoF, and thus, can completely span the operational space, while the macro system is the MP. According to results introduced in [16], the effective mass (inertia) characterizing the MHI is smaller than or equal to the one associated with the HD considered alone. In other terms, the global reflected inertia perceived by the user while using an $\mathrm{MHI}$ is, at worst, the same as the HD was grounded.

\section{B. Z-Width}

One of most important measures of performance for forcefeedback devices is the $Z$-width, i.e., the dynamic range of achievable impedances, which satisfy stability properties such as passivity [13]. In order to study the $Z$-width for an MHI, we briefly report some considerations about transparency, which have been addressed in [9]. The dynamics of the system shown in Fig. 5 can be represented by the following equations, written in the Laplace domain:

$$
\left\{\begin{array}{l}
m_{M} s^{2} x_{M}=\tau_{M}-b_{M} s x_{M}+b_{H} s\left(x_{H}-x_{M}\right)-\tau_{H} \\
m_{H} s^{2} x_{H}=\tau_{H}-b_{H} s\left(x_{H}-x_{M}\right)-f_{\mathrm{HO}} .
\end{array}\right.
$$

Relationship (5) shows that the dynamical influence of the MP on the force rendered to the user is only due to the viscous coupling $b_{H}$ between the two subsystems. However, the coefficient $b_{H}$ represents the joint damping of the HD. It is worth noticing that common impedance devices are designed in order to feature high mechanical backdriveability, which, in turn, reflects on making $b_{H}$ as small as possible. Henceforth, we will assume that $b_{H} \approx 0$ (the interested reader is referred to [9] for further details).

Given the impedance $Z_{E}$ of the VE, the force to be rendered by the HD is computed as

$$
\tau_{H}=Z_{E} x_{H}
$$

Combining the previous equation with (5), the impedance $Z^{*}$ felt by the user can be approximated as:

$$
\begin{aligned}
Z^{*}(s) & =\frac{f_{\mathrm{HO}}(s)}{x_{H}(s)} \\
& \approx \frac{\tau_{H}-s^{2} m_{H} x_{H}}{x_{H}} \\
& =Z_{E}(s)-s^{2} m_{H}
\end{aligned}
$$

which shows that as long as the HD joint damping $b_{H}$ is neglectable, dynamics of MP does not affect the impedance $Z^{*}$ felt by the user [9].

In order to evaluate the maximum operator's force that the MHI can counteract during a virtual contact, let us suppose that the end-effector is held at dynamical equilibrium by operator's forces and virtual reaction forces, i.e., $\ddot{x}_{H}=0$. This leads to cancel also the term $s^{2} m_{2} x_{H}$ from relationship (5); hence, the maximum force $f_{\max }$ can be easily computed as:

$$
f_{\max }=\max \left\{\left\|f_{\mathrm{HO}}\right\|\right\}=\max \left\{\left\|\tau_{H}\right\|\right\} .
$$


Equation (7) shows that the maximum force that can be exerted by the MHI is equal to the maximum force that the HD alone is capable to render, independently from the dynamics of the MP.

Summarizing, if the damping term $b_{H}$ of the HD mounted on the MP is neglectable, the $Z$-width of an MHI is directly inherited from the HD itself and does not depend on the MP dynamics.

\section{Motion PERformance}

Results reported so far show that it is generally reasonable to consider specification (4a) to be true. The same cannot, however, be said for (4b), which is verified only if the HD end-effector does not reach its workspace boundary. Hence, denoting the size of the HD workspace with $2 \Delta_{\text {WS }}$, spurious forces are avoided if the tracking error $e$ is kept below $\Delta_{\mathrm{WS}}$ :

$$
f_{\mathrm{WS}}(t)=0 \Longleftrightarrow|e(t)|<\Delta_{\mathrm{WS}} \quad \forall t .
$$

The previous condition naturally reflects on the input signal $x_{H}$ since it is related to the error signal through the relationship (see Fig. 4)

$$
e(s)=\frac{1}{1+C(s) H(s)} x_{H}(s)=G(s) x_{H}(s)
$$

where $G(s)$ is the error transfer function. Henceforth, we will consider an input signal $x_{H}$ as correctly tracked by the MHI when the corresponding tracking error $e(t)$ satisfies relationship (8). It is worth remarking that in the following analysis, the emphasis is naturally put on the maximum tracking error $e_{\max }$. In particular, the only requirement on the steady-state value $e(\infty)$ is to be smaller than the workspace dimension $\Delta_{\mathrm{Ws}}$.

In order to come up with analytic conditions on the input $x_{H}(t)$, we restrict our study to three main classes of signals, whose combination resembles standard operator movements: step displacements, ramp displacements, and sinusoidal displacements. Clearly, this approach does not cover all possible scenarios, since $x_{H}(t)$ is generated by the operator's hand. However, it provides useful and easy-to-test guidelines on how to design MHI controller given knowledge of the specific application in which the MHI will be used (and thus of input signals $x_{H}(t)$ to be expected).

\section{A. Step Displacement}

In this section, we draw preliminary observations about the maximum amplitude $A_{u}$ of an ideal step displacement defined as

$$
x_{H}(t)=A_{u} t, \quad t \geq 0
$$

for which the end-effector never reaches its workspace boundary during tracking. It is well known that in second-order stable systems, the step response envelope is monotonically decreasing, so the maximum error occurs at $t=0^{+}$. Hence, the maximum amplitude of an ideal step position signal applied to an MHI must be such that:

$$
A_{u}<\Delta_{\mathrm{WS}} \cdot(\text { Ideal step limitation })
$$

\section{B. Ramp Displacement}

In this section, ramp input signals are analyzed. The target is to evaluate what is the maximum slope of a ramp signal that can be correctly tracked by the MHI. Let us consider an ideal ramp $x_{H}=V_{R} t$. Since $H(s)$ has one pole at zero, the MHI tracks the input with a finite steady-state error

$$
e(\infty)=\lim _{s \rightarrow 0} s e(s)=\frac{V_{R}}{K_{v}}
$$

where the velocity gain $K_{v}$ is given by

$$
K_{v}=\lim _{s \rightarrow 0} s C(s) H(s)=\frac{k_{M} K_{p}}{b_{M}} .
$$

The maximum error $e_{\max }$ can be reached during the transient, and, in this case, it can be computed from the overshoot $e_{o}$ of the system

$$
e_{o} \triangleq \frac{e_{\max }-e(\infty)}{e(\infty)} .
$$

Note that $e_{o}$ does not depend on the slope of the ramp, but only on $H(s)$ and $C(s)$. Clearly, if the tracking error has no overshoot peak, $e_{\max }=e(\infty)$, and the previous equation becomes $e_{o}=0$. By combining (11) and (12), it follows

$$
e_{\max }=\left(1+e_{o}\right) \frac{V_{R}}{K_{v}}
$$

which holds also in case of $e_{o}=0$. The maximum error $e_{\max }$ is proportional to the ramp slope $V_{R}$ and can be computed by experimentally determining the system overshoot $e_{o}$ for a given MP. Finally, in order for $e_{\max }<\Delta_{\mathrm{WS}}$ to be true, the input signal must be such that

$$
V_{R}<\frac{\Delta_{\mathrm{WS}} K_{v}}{\left(1+e_{o}\right)} . \text { (Slope limitation) }
$$

Note that such limitation holds no matter what is the response to the system to a ramp input (overshoot or not).

\section{Human-Made Step Displacement}

When the HD end-effector is driven by a human operator, it features finite velocities and accelerations (see Fig. 6). A possible way to analyze this kind of movements is to approximate human-made steps by a continuous, piece-wise linear signal (see the right side of Fig. 6). An ideal step of amplitude $A_{u}$ can thus be replaced by

$$
x_{H}(t)= \begin{cases}V_{r} t, & 0 \leq t \leq T \\ V_{r} T, & t>T\end{cases}
$$

where the ramp slope $V_{r}$ and the duration $T$ are such that $V_{r} T=A_{u}$. Notice that this approximation is still an abstraction of real step movements, since it assumes infinite acceleration capability.

Previous results obtained in case of ramp displacements can be exploited in order to analyze this kind of reference signal. Given the workspace limit $\Delta_{\mathrm{WS}}$, a human-made step movement will or will not drive the end-effector to the workspace boundary, depending on the slope $V_{r}$ and on its duration $T$, yielding the human-made step amplitude $V_{r} T$. As pointed out earlier, the maximum tracking error grows proportionally with the system 


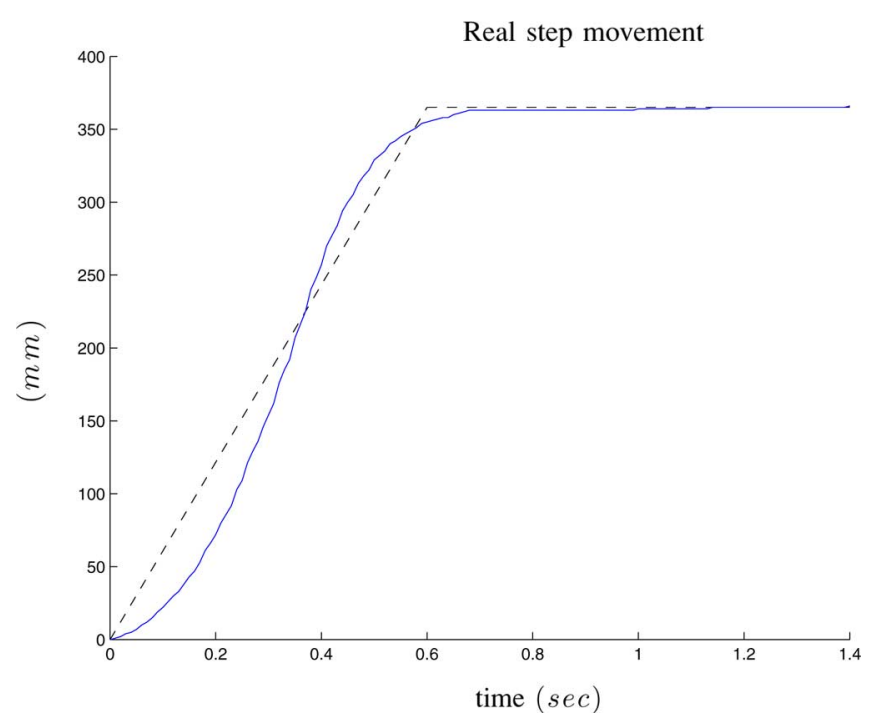

Fig. 6. Example of user-made step movement (solid line) and its piecewiselinear approximation (dashed).

overshoot $e_{o}$. Thus, a reasonable choice is to design the controller such that $e_{o}=0$. In the next section, it will be shown that this behavior can be achieved by a suitable choice of the controller parameters, which leads to real poles for closed-loop error transfer function. In this case, the maximum tracking error corresponds to the steady-state value $V_{r} / K_{v}$. Obviously, if the endeffector velocity $V_{r}$ is such that $V_{r} / K_{v}<\Delta_{\mathrm{WS}}$, then the MP is able to track steps of any amplitude $A_{u}$ without loosing transparency. Now, let us focus on the more interesting case $V_{r}>$ $K_{v} \Delta_{\text {WS }}$. The tracking error may exceed $\Delta_{\text {WS }}$ depending on the duration $T$. Intuitively, the system can tolerate rapid movements provided that they do not last too long. In other words, large displacements must be executed at reduced velocity. To quantify the earlier observations, let us study the maximum step amplitude $A_{u}$ giving rise to a tracking error smaller than $\Delta_{\mathrm{WS}}$, as a function of $V_{r}$. In case of real poles $p_{1}<p_{2}<0$, in response to the reference $x_{H}(t)$, the time evolution of the tracking error is

$$
e(t)=A+B e^{p_{1} t}+C e^{p_{2} t}, \quad 0 \leq t \leq T
$$

while $|e(t)|<|e(T)|$ for $t>T$. If one neglects the fastest dynamics, the error can be approximated as

$$
e(t) \simeq A+C e^{p_{2} t}, \quad 0 \leq t \leq T
$$

where

$$
\begin{aligned}
A & =\frac{V_{r}}{K_{v}} \\
C & =\frac{\left(1 / \tau+p_{2}\right) V_{r}}{p_{2}\left(p_{2}-p_{1}\right)}
\end{aligned}
$$

and $\tau=m_{M} / b_{M}$. At this point, for a fixed $V_{r}$, it is possible to find the maximum time $T$ such that the error remains below $\Delta_{\text {WS }}$ :

$$
\max _{T: e(T)<\Delta_{\mathrm{wS}}} T \simeq \frac{1}{p_{2}} \log \left(\frac{\Delta_{\mathrm{WS}}-A}{C}\right) .
$$

Equivalently, this allows to compute the maximum displacement $A_{u}$, executed at velocity $V_{r}$, which does not lead to reach

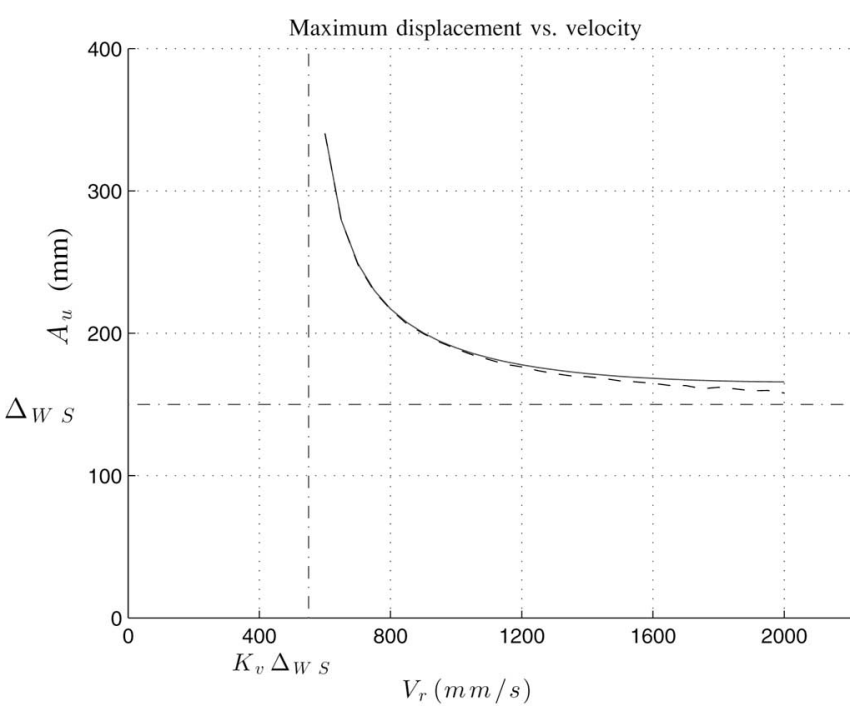

Fig. 7. Maximum admissible step amplitude: actual (solid line) and approximated (dashed line) amplitude.

the WS boundary:

$$
A_{u} \simeq \frac{V_{r}}{p_{2}} \log \left(\frac{\Delta_{\mathrm{WS}}-V_{r} / K_{v}}{\left[\left(1 / \tau+p_{2}\right) / p_{2}\left(p_{2}-p_{1}\right)\right] V_{r}}\right) .
$$

In Fig. 7, the approximated maximum amplitude $A_{u}$ (dashed line) is depicted for different slopes $V_{r}$, and it is compared to the actual value (solid line) obtained from simulations. It turns out that approximation (14) is quite accurate, at least as long as $V_{r}$ does not grow excessively (in that case, the maximum $T$ is very small and the fastest mode cannot be neglected). Moreover, the results are in good agreement with the intuition. When $V_{r}$ slows down to $K_{v} \Delta_{\text {WS }}$ (which represents the maximum velocity of correctly trackable ramp signals), the maximum displacement $A_{u}$ can be arbitrarily large. Conversely, when $V_{r}$ increases, the amplitude of the tolerable displacements decreases, and, in the limit, it shrinks to $\Delta_{\mathrm{WS}}$ (which represents the limitation for the ideal step).

\section{Sinusoidal Displacement}

In this section, we study the maximum amplitude/frequency of a sinusoidal input signal $x_{H}=A_{s} \sin (\omega t)$ that can be correctly tracked by the MHI. Given the linearity of the overall MHI system, at steady state, we have

$$
e(t)=A_{s}\|G(j \omega)\| \sin (\omega t+\angle(G(j \omega))) .
$$

In order for $|e(t)|<\Delta_{\mathrm{WS}}$, the sinusoid amplitude $A_{s}$ and frequency $\omega$ must satisfy:

$$
A_{s}\|G(j \omega)\|<\Delta_{\mathrm{ws}}
$$

Since $H(s)$ typically has a low-pass filter behavior, $G(s)$, in turn, is a high-pass system; hence, higher frequency sinusoids must have lower amplitude, and vice-versa. It is possible to define a region $\mathcal{I}$ of the $\left(\omega, A_{s}\right)$ plane

$$
\mathcal{I}=\left\{\left(\omega, A_{s}\right): A_{s}\|G(j \omega)\|<\Delta_{\mathrm{WS}}\right\}
$$


whose boundary

$$
\gamma: A_{s}\|G(j \omega)\|=\Delta_{\mathrm{WS}}
$$

can be numerically computed. Then, region $\mathcal{I}$ represents all the sinusoidal inputs that can be correctly rendered by an MHI:

$$
\left(\omega, A_{s}\right) \in \mathcal{I} .(\text { Sinusoidal limitation })
$$

Note that the previous analysis only applies to the steady-state behavior of the system.

\section{DESIGN OF MHI Motion CONTROLlER}

In this section, we use the indicators previously introduced to analytically compute the controller parameters $K_{p}$ and $K_{d}$, in order to guarantee the stability and the boundedness of tracking error within workspace limits. The analysis is divided into three steps, corresponding to the three classes of signals previously introduced.

\section{A. Ideal Step Response Specifications}

Since the system stability is preserved for any positive value of the controller parameters $K_{p}, K_{d}$, the maximum tracking error is still attained at $t=0^{+}$and does not depend on the choice of $K_{p}, K_{d}$.

\section{B. Ramp Response Specifications}

In order to track a ramp with slope $V_{R}$ given a prescribed $\Delta_{\mathrm{WS}}$, the controller parameters can be determined as follows. First, note that $e_{\max } \geq e(\infty)$. Thus, a slope $V_{R}$ can be correctly tracked only if

$$
\frac{V_{R}}{K_{v}} \leq \Delta_{\mathrm{WS}}
$$

Recalling the expression of the velocity gain $K_{v}$, the latter inequality yields a lower bound on the proportional gain

$$
K_{p} \geq \frac{b_{M}}{\Delta_{W S} k_{M}} V_{R} .
$$

Once a suitable value has been set for $K_{p}$ according to (16), a proper choice of the derivative gain $K_{d}$ can prevent the error signal $e(t)$ from exhibiting overshoot, thus ensuring $e_{\max }=$ $e(\infty)$. After some computation (presented in Appendix A), it turns out that the error transfer function $G(s)$ has complex poles if and only if

$$
\Delta=\alpha_{0}+\alpha_{1} K_{d}^{2}+\alpha_{2} K_{d}-4 \alpha_{3} K_{p}<0
$$

where $\alpha_{i}$ are positive coefficients that only depend on the parameters of the mobile robot. The equation $\Delta=0$ represents a parabola in the plane $\left(K_{d}, K_{p}\right)$, as shown in Fig. 8. Hence, a necessary condition in order to avoid overshoot is that $\Delta \geq 0$. However, even in case of real poles, the error signal can still exhibit overshoot for some choice of the controller gains. These values can be analytically determined (see Appendix A) as those

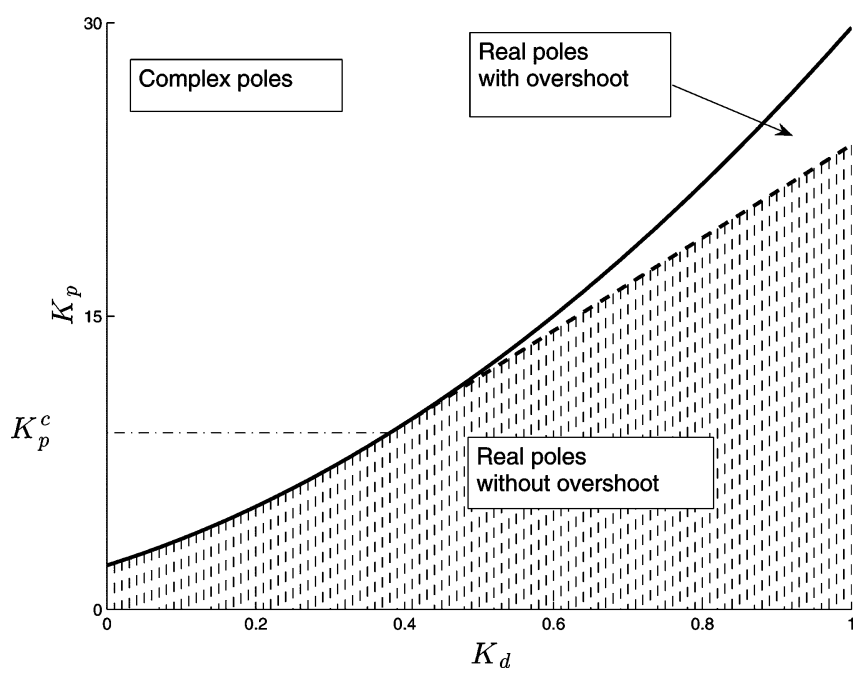

Fig. 8. Controller design for ramp response: parabola $\Delta=0$ (solid line), line $K_{d}=\tau K_{p}$ (dashed line). The shaded region represents the values $\left(K_{p}, K_{d}\right)$ ensuring no overshoot.

satisfying the following relations

$$
\begin{aligned}
\Delta & >0 & & (\text { Real poles }) \\
K_{p} & >K_{p}^{c} & & (\text { Possible overshoot }) \\
K_{p} & >\frac{K_{d}}{\tau} & & \text { (Overshoot) }
\end{aligned}
$$

where $K_{p}^{c}=b_{M}^{2} / k_{M} m_{M}$, and $\tau=m_{M} / b_{M}$ denotes the time constant of the MP. Hence, there exist real poles giving rise to overshoot if $K_{p}>K_{p}^{c}$ and $\left(K_{p}, K_{d}\right)$ are between the line $K_{p}=K_{d} / \tau$ and the parabola $\Delta=0$ (see Fig. 8). Thus, from (17)-(20), it follows that the error signal does not have overshoot if and only if the pair $\left(K_{p}, K_{d}\right)$ is such that $\Delta>0$, and either

$$
K_{p} \leq K_{p}^{c}
$$

or

$$
K_{p} \leq \frac{K_{d}}{\tau}
$$

The values $\left(K_{p}, K_{d}\right)$, which ensure that no overshoot shows up are depicted in Fig. 8 (shaded region), for a Nomad-like MP model. Note that the boundaries of such a region can be easily computed and only depend on the parameters $\left(m_{M}, b_{M}, k_{M}\right)$ of the MP. At this point, the design of a controller guaranteeing $e_{\max }<\Delta_{\mathrm{WS}}$ for a given ramp $V_{R}$ can be summarized as follows:

1) choose a value $K_{p}^{*}$ such that (16) is satisfied;

2) if $K_{p}^{*} \leq K_{p}^{c}$, choose a value $K_{d}^{*}$ such that $\Delta>0$; and

3) if $K_{p}^{*}>K_{p}^{c}$, choose a value $K_{d}^{*}$ such that $K_{d}^{*} \geq \tau K_{p}^{*}$.

The aforementioned procedure has a simple graphical interpretation. For the chosen $K_{p}^{*}$ according to (16):

1) if $K_{p}^{*} \leq K_{p}^{c}$, select a value for $K_{d}^{*}$ such that the pair $\left(K_{p}^{*}, K_{d}^{*}\right)$ lies below the parabola $\Delta=0$ (solid line in Fig. 8) and

2) if $K_{p}^{*}>K_{p}^{c}$, select a value for $K_{d}^{*}$ such that the pair $\left(K_{p}^{*}, K_{d}^{*}\right)$ lies below the line $K_{d}=\tau K_{p}$ (dashed line in Fig. 8). 
Clearly, the controller designed for a given $\bar{V}_{R}$ ensures $e_{\max }<\Delta_{\mathrm{WS}}$ for all slopes $V_{R} \leq \bar{V}_{R}$.

\section{Sinusoidal Specifications}

In order to design an MHI that is capable of tracking a sinusoidal reference $\left(\omega, A_{s}\right)$ without exceeding the workspace, the controller parameters must be chosen such that

$$
\|G(j \omega)\| \leq \frac{\Delta_{\mathrm{WS}}}{A s} .
$$

For this purpose, let us study the dependency of $\|G(j \omega)\|$ on $\left(K_{p}, K_{d}\right)$. It can be shown that

$$
\|G(j \omega)\|=\frac{\sqrt{\beta_{0}}}{\sqrt{\beta_{0}+\beta_{1} K_{p}^{2}+\beta_{2} K_{d}^{2}-\beta_{3} K_{p}+\beta_{4} K_{d}}}
$$

where $\beta_{i}$ are positive functions of $\omega$ and of the robot parameters. Denoting by $\mathcal{E}\left(K_{p}, K_{d}\right)$ the argument of the square root at the denominator, the required parameters $\left(K_{p}, K_{d}\right)$ are those satisfying

$$
\mathcal{E}\left(K_{p}, K_{d}\right) \geq \beta_{0} \frac{A_{s}^{2}}{\Delta_{\mathrm{WS}}^{2}} \triangleq C .
$$

Note that the curve $\mathcal{E}\left(K_{p}, K_{d}\right)=C$ represents an ellipse in the $\left(K_{p}, K_{d}\right)$ plane, whose center and radii can be analytically computed from the coefficients $\beta_{i}$ and the desired value $C$ (the reader is referred to Appendix B for the mathematical details). From a geometrical viewpoint, inequality (22) states that all the parameter values ensuring $e_{\max }<\Delta_{\mathrm{WS}}$ are those lying outside the ellipse $\mathcal{E}\left(K_{p}, K_{d}\right)=C$. By tracing all the ellipses $\mathcal{E}_{i}$ corresponding to a set of sinusoids $\left(w_{i}, A_{s_{i}}\right)$ at different frequencies and amplitudes, it is possible to define the region $\mathcal{F}$ in the $\left(K_{p}, K_{d}\right)$ plane defined as

$$
\mathcal{F}=\bigcup_{i} \mathcal{E}_{i}
$$

In order for the MHI to correctly track the chosen set of sinusoids, the controller parameters $\left(K_{p}, K_{d}\right)$ must lie outside $\mathcal{F}$.

Note that although design of PD controllers have been separately studied for ramps and sinusoids, it is possible to choose the pair $\left(K_{p}, K_{d}\right)$ that matches both project criteria, thus simultaneously guaranteeing performance specifications with respect to ramps and sinusoids. Actually, one should also consider that desired performance specifications could lead to values of parameter resulting in unfeasible control laws, due to system mechanical limitations.

\section{BOUNDED ACCELERATION}

In this section, we consider a more realistic scenario, which takes into account some physical limitations of the MP. Specifically, it is assumed that the MP can accelerate up to a maximum value $a_{\operatorname{MAX}}$ (e.g., due to actuator saturation). The following analysis aims at assessing the MHI performance in the presence of bounded acceleration. To this purpose, the controller is supposed to be purely proportional (i.e., $K_{d}=0$ in (1)), and the considered reference signal is a ramp $x_{H}(t)=V_{r} t$. Fig. 9
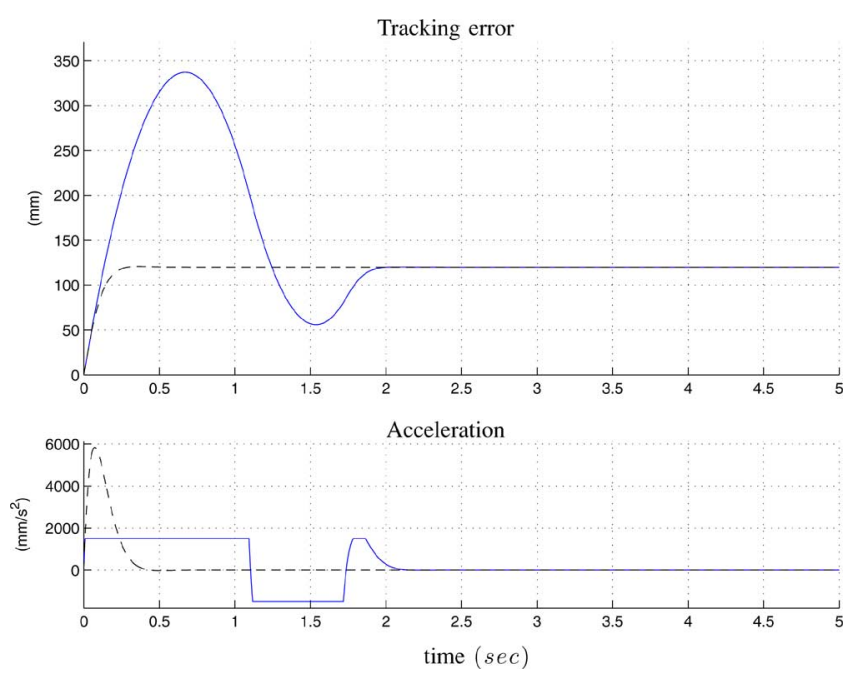

Fig. 9. Effects of bounded acceleration for a Nomad-like MP, with $V_{r}=$ $1000 \mathrm{~mm} / \mathrm{s}$ and $a_{\mathrm{MAX}}=1500 \mathrm{~mm} / \mathrm{s}^{2}$. Comparison of tracking error (top) and acceleration signal (bottom) for saturated model (solid line) and linear model (dashed line).

shows the typical effect of bounded acceleration on the tracking error. When the operator's movements are too fast (i.e., large $V_{r}$ ) and/or the controller gain $K_{p}$ is too large, the driving signal may subject the MP to unfeasible accelerations. As a result, the actual maximum tracking error becomes much larger than that predicted by the nominal linear model. In the remaining of the section, we will try to evaluate the performance degradation in case of acceleration constraints: given $a_{\mathrm{MAX}}$ and $V_{r}$, we will compare the tracking errors with or without saturation, as a function of the controller gain. Hereafter, the main results are presented, while the mathematical details can be found in Appendix C.

Let us denote by $W(s)$ the closed-loop transfer function from the reference signal $x_{H}$ to the MP position $x_{M}$ :

$$
W(s)=\frac{C(s) H(s)}{1+C(s) H(s)}=\frac{\omega_{n}^{2}}{s^{2}+2 \zeta \omega_{n} s+\omega_{n}^{2}}
$$

where $\omega_{n}=\sqrt{k_{M} K_{p} / m_{M}}$ is the natural frequency and $\zeta=$ $b_{M} / 2 \sqrt{m_{M} k_{M} K_{p}}$ is the damping ratio of the poles. Depending on the choice of the proportional gain $K_{p}$, the poles can be complex, real-coincident, or real-distinct. However, as already pointed out in Section VI, upper bounds on the steady-state tracking error reflect on lower bounds on $K_{p}$. For this reason, in the following, we assume

$$
K_{p}>\frac{b_{M}^{2}}{4 m_{M} k_{M}}
$$

so that $W(s)$ has complex poles. Note that constraint (16) implies $K_{p}>b_{M}^{2} / 4 m_{M} k_{M}$ whenever $V_{r} / \Delta_{\mathrm{WS}}>b / 4 m_{M}$.

According to the linear model, straightforward computations show that when neglecting saturation, the maximum tracking error is

$$
e_{\max }=\left(2 \zeta+e^{-(\pi-\phi) / \tan (\phi)}\right) \frac{V_{r}}{\omega_{n}}
$$


where $\phi=\arccos (\zeta) \in(0, \pi / 2)$. Notice that $e_{\max }$ could be made arbitrarily small by choosing a sufficiently large $K_{p}$. In the limit, when $K_{p} \rightarrow \infty, e_{\max } \rightarrow 0$. This observation suggests that assumption (24) is not so stringent.

Clearly, small $e_{\max }$ necessarily requires high accelerations. Suppose now that the maximum feasible acceleration is $a_{\text {MAX }}$. First, one may wonder whether acceleration ever saturates. Given the ramp slope $V_{r}$ and the acceleration constraint $a_{\mathrm{MAX}}$, this depends on the value of $K_{p}$. It turns out that the quantity $\omega_{n} V_{r} / a_{\text {MAX }}$ plays a crucial role in determining the saturation activation.

1) Conditions on saturation activation

a) If $\omega_{n} V_{r} / a_{\mathrm{MAX}}<1$, then saturation never occurs.

b) Conversely, if $\omega_{n} V_{r} / a_{\mathrm{MAX}}>e$, then surely there exists a time $t_{i}$ when saturation begins.

c) Finally, if $1<\omega_{n} V_{r} / a_{\mathrm{MAX}}<e$, saturation may occur or may not, depending on the value of the damping ratio $\zeta$. Specifically, let $\phi^{*} \in(0, \pi / 2)$ be the (unique) solution of

$$
\phi=\log \left(\frac{\omega_{n} V_{r}}{a_{\mathrm{MAX}}}\right) \tan (\phi) .
$$

Then, saturation occurs if and only if $\phi>\phi^{*}$, or equivalently, if and only if $\zeta<\zeta^{*}$, where $\zeta^{*}=$ $\cos \left(\phi^{*}\right)$.

Let us suppose that the chosen $K_{p}$ leads to saturation activation, the following upper and lower bounds on the maximum tracking error can be established.

2) Bounds on the maximum tracking error

$$
\frac{1}{2} \frac{V_{r}^{2}}{a_{\mathrm{MAX}}}<e_{\max }<f\left(K_{p}, V_{r}, a_{\mathrm{MAX}}\right)
$$

where

$$
\begin{aligned}
f\left(K_{p},\right. & \left.V_{r}, a_{\mathrm{MAX}}\right) \\
= & \frac{2 \zeta}{\omega_{n}} V_{r}+\frac{a_{\mathrm{MAX}}}{\omega_{n}^{2}} \\
& \times\left(\frac{1}{2}\left(\sqrt{\left(\frac{\omega_{n} V_{r}}{a_{\mathrm{MAX}}}\right)^{2}-\left(1-\zeta^{2}\right)}-\zeta\right)^{2}-2 \zeta^{2}+1\right) .
\end{aligned}
$$

It is interesting to note that the aforementioned bounds are asymptotically tight, in the sense that

$$
\lim _{K_{p} \rightarrow \infty} f\left(K_{p}, V_{r}, a_{\mathrm{MAX}}\right)=\frac{1}{2} \frac{V_{r}^{2}}{a_{\mathrm{MAX}}}
$$

which, in turns, implies

$$
\lim _{K_{p} \rightarrow \infty} e_{\max }=\frac{1}{2} \frac{V_{r}^{2}}{a_{\mathrm{MAX}}} .
$$

Previous results are illustrated in Fig. 10, where the maximum tracking error for a linear (dashed line) and a saturated (solid line) model are compared, for different values of $K_{p}$. For convenience, the quantity reported on the abscissa is $\omega_{n} V_{r} / a_{\mathrm{MAX}}$.

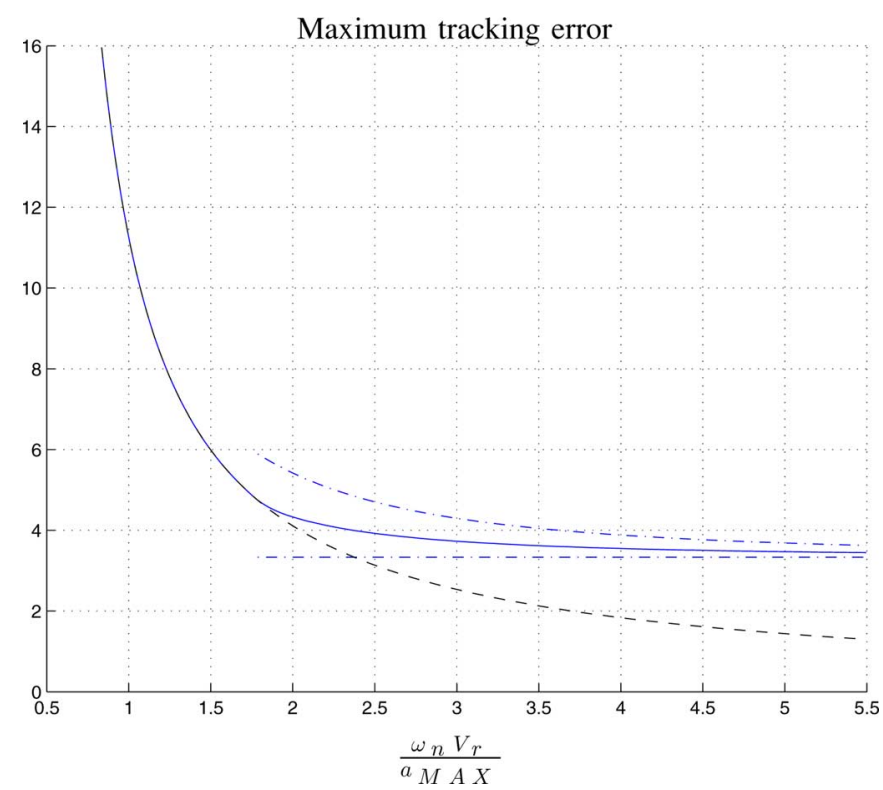

Fig. 10. Maximum tracking error: saturated model (solid), linear model (dashed), and bounds (dash-dotted).

Note that, being $V_{r}$ and $a_{\mathrm{MAX}}$ fixed, this choice corresponds to scale the $x$-axis. For small $K_{p}$, acceleration does not saturate, and the maximum errors coincide. When $K_{p}$ is such that the damping ratio falls below the threshold $\zeta^{*}$, saturation occurs, and the maximum tracking error decreases more slowly than it does in the linear case. The main difference between the two error signals relies on the asymptotic behavior. Differently from what happens in the linear case, when $K_{p}$ tends to infinity, the maximum tracking error does not vanish. The lower bound $V_{r}^{2} / 2 a_{\mathrm{MAX}}$ can be thought of as an intrinsic limitation imposed by the acceleration bound, no matter which value of $K_{p}$ is chosen. For instance, with the proportional controller considered here, there is no chance to avoid the HD workspace boundary $\Delta_{\mathrm{WS}}$ if the speed of the reference signal is bigger than $\sqrt{2 a_{\mathrm{MAX}} \Delta_{\mathrm{WS}}}$. Conversely, the upper bound $f\left(K_{p}, V_{r}, a_{\mathrm{MAX}}\right)$ can be exploited in order to compute the minimum $K_{p}$ ensuring a tracking error below a given $\Delta_{\mathrm{Ws}}$. However, high values of $K_{p}$ also have drawbacks, the most annoying one being the generation of very low damped oscillations. Thus, the value of the proportional gain has to be chosen by trading off maximum tracking error and settling time. Another possibility is to adopt a control scheme with more DoFs, as we did in the previous sections. It turns out that, also in this case, an additional derivative term can be effective in damping undesired oscillations, without excessively increasing the error peak.

We conclude this section, with a remark on possible bounds also on the feasible MP velocities. It can be easily shown that until the tracking error does not attain its maximum value, the velocity signal resulting from the saturated model is always less than the reference velocity $V_{r}$ and reaches $V_{r}$ when $e(t)=e_{\max }$. As a consequence, if $V_{r}$ is smaller than the maximum MP velocity (which is trivially a necessary condition for the tracking error to remain bounded), the previous analysis still holds, as far as the maximum error is concerned. Velocity bounds only affect 

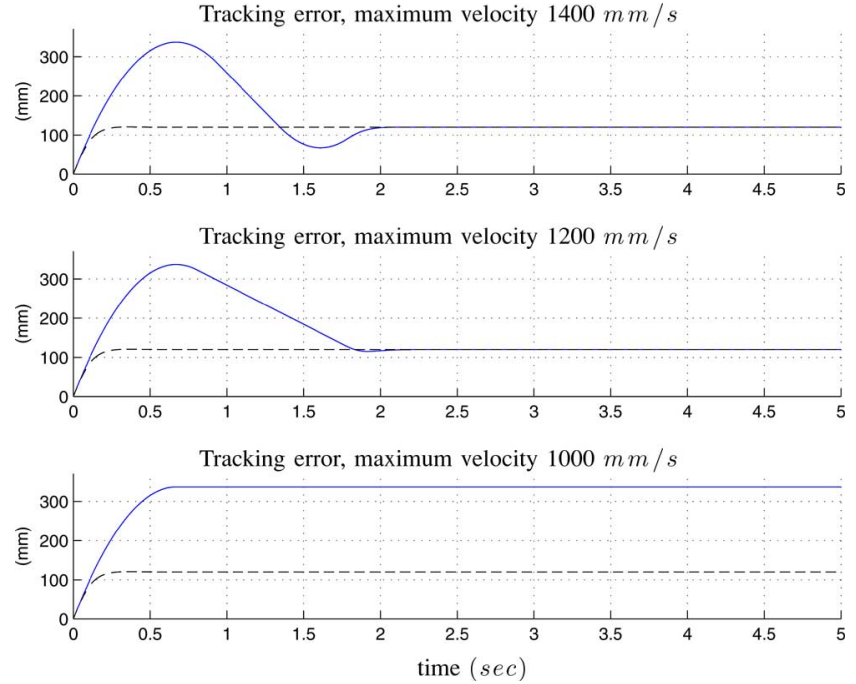

Fig. 11. Effects of bounded acceleration and bounded velocity for a Nomadlike MP, with $V_{r}=1000 \mathrm{~mm} / \mathrm{s}$ and $a_{\mathrm{MAX}}=1500 \mathrm{~mm} / \mathrm{s}^{2}$. Comparison of tracking error for saturated model (solid line) and linear model (dashed line).

the convergence rate of the error to its steady-state value. Qualitatively, smaller difference between maximum velocity and $V_{r}$ leads to the slower convergence. In the limit, if the maximum velocity equals $V_{r}$, the tracking error remains constant to its maximum value (see Fig. 11).

\section{EXPERIMENTAL VALIDATION}

The theoretical analysis described in Section $\mathrm{V}$ builds on the linear model chosen for the MP, allowing us to come up with analytical relationships between performance indicators and model parameters. In order to verify to which extent the model fits to real system in predicting workspace boundary outreaching, an experimental campaign has been conducted using an $\mathrm{MHI}$ composed of a PHANToM Premium 1.5 haptic interface and a Nomad XR4000 MP [10]. The latter is an omni-directional robot, featuring high levels of inertia. All the experiments have been performed along a single DoF, and, for each trial, the actual tracking error has been compared to the one predicted by the corresponding model. As pointed out in Section II, the tracking error $e$ corresponds to the displacement of the end-effector with respect to the center of its workspace, thus being directly available from the readings of the haptic interface encoders.

A preliminary identification phase was required before experimental validation. Several sets of input-output data $\left\{x_{r}, x_{M}\right\}$, corresponding to different classes of input signals (e.g., square waves, ramps, and sinusoids) have been collected. Then, the values of the model parameters $k$ and $b$ have been tuned by comparing the actual and simulated outputs. Note that the total mass $m$ was known a priori from the technical specifications of the employed devices. [11].

The values of the controller parameters $K_{p}$ and $K_{d}$ have been selected according to the procedure described in Section VI. In the design stage, for safety reasons, the HD was supposed to have a workspace smaller $\left(\Delta_{\mathrm{WS}}=150 \mathrm{~mm}\right)$ than the actual one. As a matter of fact, this choice allows one to excite the system also with inputs leading to tracking errors greater than

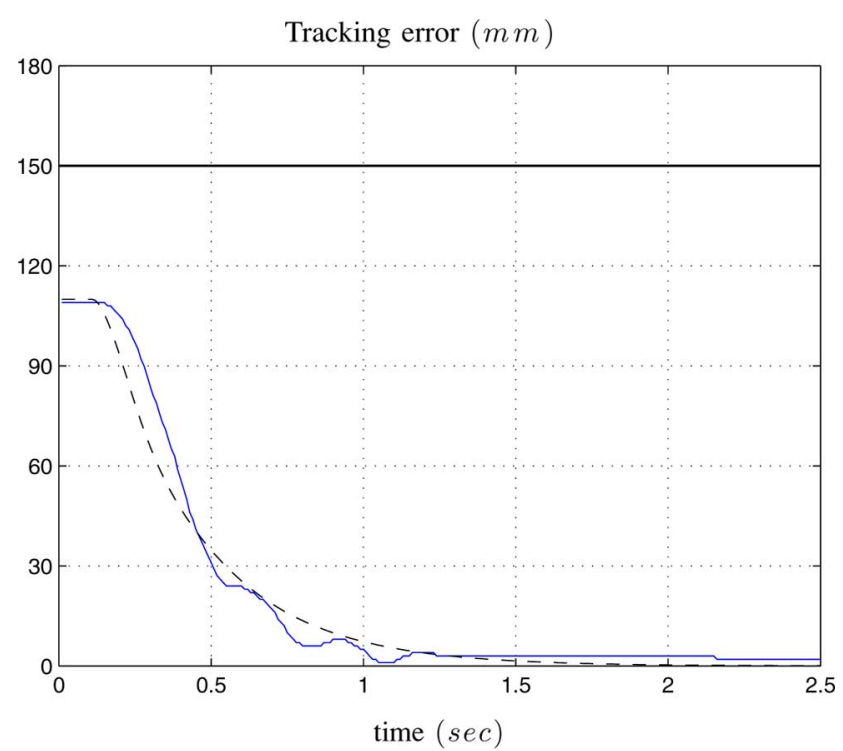

Fig. 12. Tracking error of a step input $x_{H}(t)=100$ : actual (solid line) and predicted by the model (dashed). Thick solid line represents the HD workspace limit which is $150 \mathrm{~mm}$.

$\Delta_{\mathrm{WS}}$, without physically reaching the real workspace boundary of the HD.

Several sets of experimental trials have been carried out to validate the performance limitations derived in the previous sections.

Even if the validation of ideal step limitation (see Section VA) is straightforward, for the sake of completeness, we briefly report an experiment in which the MP was excited with an ideal step reference of amplitude $A_{u}=100 \mathrm{~mm}$. In Fig. 12, the actual (solid line) and predicted (dashed line) tracking errors are shown. Thick solid lines represent the desired maximum error, due to workspace limits. As predicted by the model, the real tracking error confirmed that the system was stable, thus guaranteeing that as long as the amplitude of the position steps is inside the workspace limit, the end-effector never reaches its maximum extension.

To evaluate ramp limitations (see Section V-B), the MHI was excited using several reference signals $x_{H}=V_{R} t$, featuring different slopes $V_{R}$. This input has been generated using a Pioneer 2DX mobile robot to move MHI end-effector at constant speed. While the MHI stood still, the driver robot was accelerated in order to reach a desired velocity $V_{R}$, and then, hooked up to the MHI end-effector (through a velcro connection), thus exciting the MHI with the desired ramp reference. Again, in Fig. 13, the predicted and actual tracking errors for the considered input signals are depicted. It can be noted that the maximum tracking error is achieved at the steady state, i.e., the controlled MHI does not exhibit overshoot. Moreover, the maximum tracking error is almost proportional to the input velocity, as predicted by the theoretical analysis.

As already discussed in Section V-C, the analysis of step limitation can be extended to human-made step input by exploiting results about ramp limitation. The finite velocities characterizing real inputs let the system begin tracking the reference before the operator has reached the steady-state value, thus 

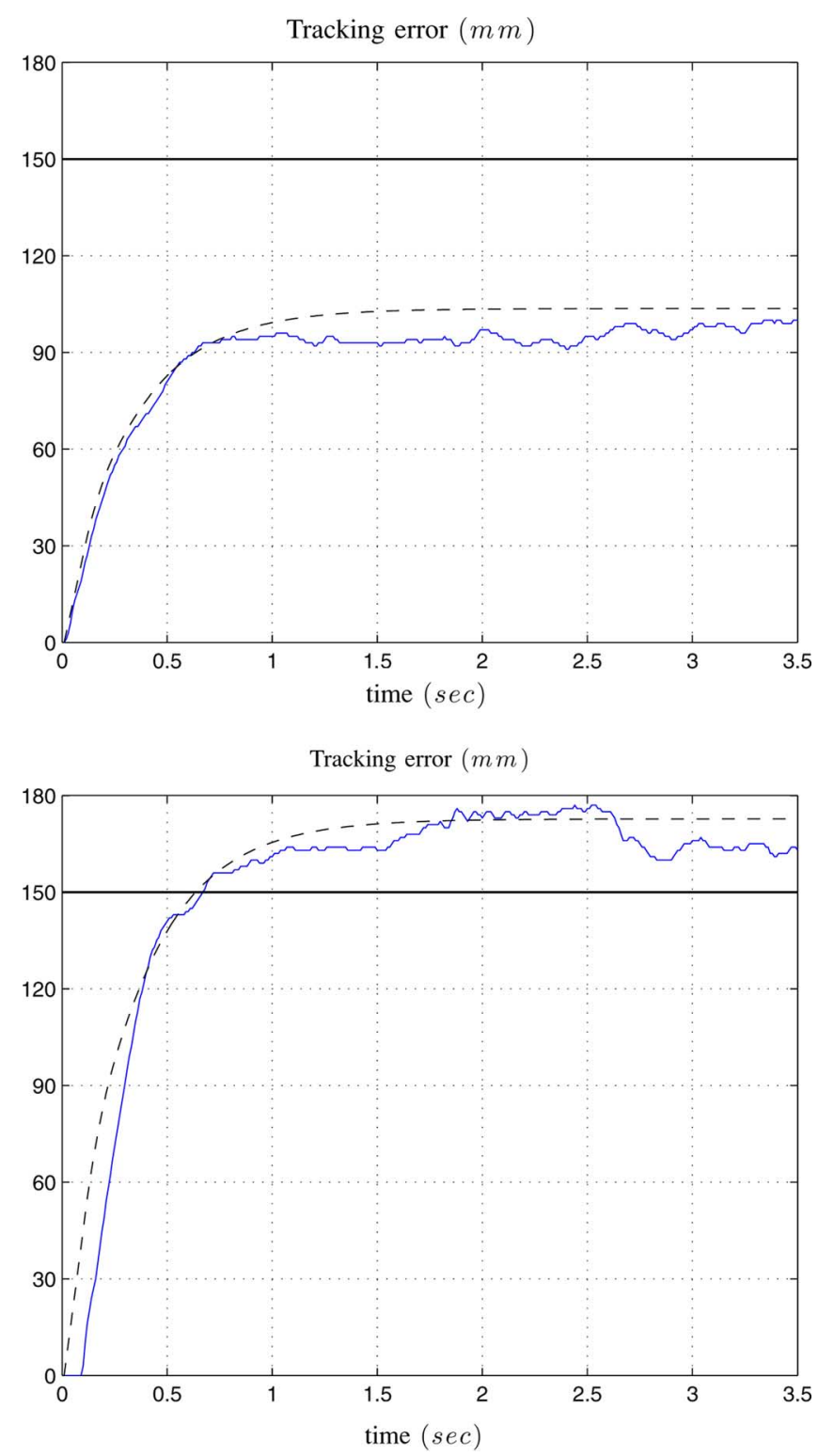

Fig. 13. Actual (solid line) and predicted (dashed line) tracking errors of ramp inputs. On the top $x_{H}(t)=300 t$, on the bottom it is $x_{H}(t)=500 t$. Thick solid line represents the HD workspace limit which is $150 \mathrm{~mm}$.

allowing larger displacements than the workspace dimension, as it was in the case of ideal step. This result has been evaluated by exciting the MHI with several human-made step displacements and comparing real tracking error with model predicted one.

In Fig. 15, we report an example of human-made step input (solid line), which can be approximated with a piecewise linear signal (dashed line), as the one shown in Fig. 6. The ramp features a slope of about $816 \mathrm{~mm} / \mathrm{s}$ and a duration of $0.25 \mathrm{~s}$, which, in turn, results in a step amplitude $A_{u}=208 \mathrm{~mm}$. Referring to Fig. 7, such values of velocity and amplitude correspond to a safe input, even if it is near to the curve of critical inputs. In other terms, the tracking error may get close to the workspace limitation, but should never reach it. To confirm the analytical prediction, Fig. 15 shows the real tracking error (solid line) and the model predicted one (dashed line) for the real human-made

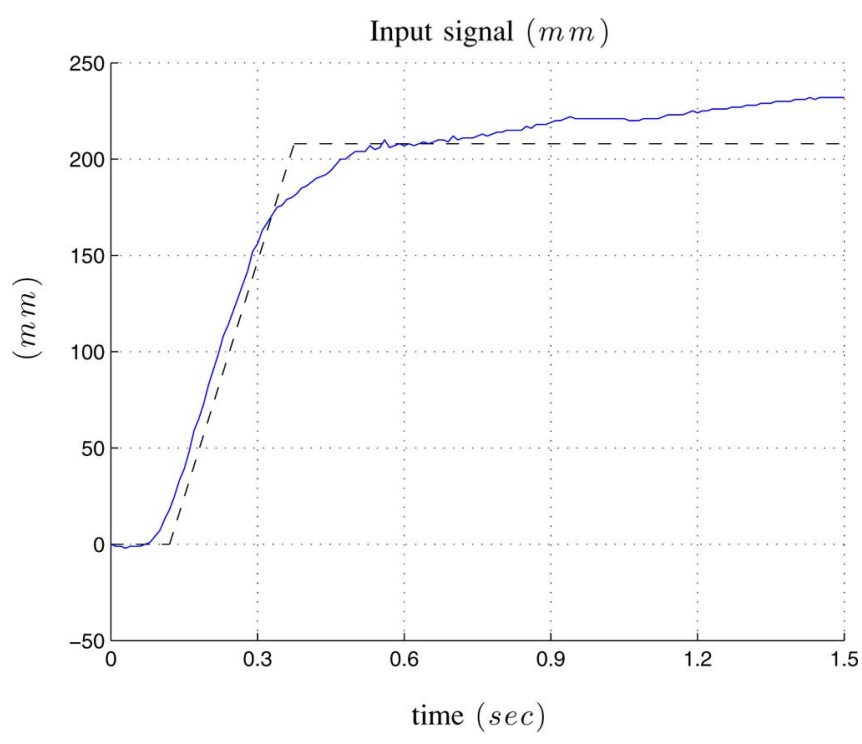

Fig. 14. Real hand-generated input (solid line), approximated by an ideal signal (dashed line). The slope of the ramp is $816 \mathrm{~mm} / \mathrm{s}$.

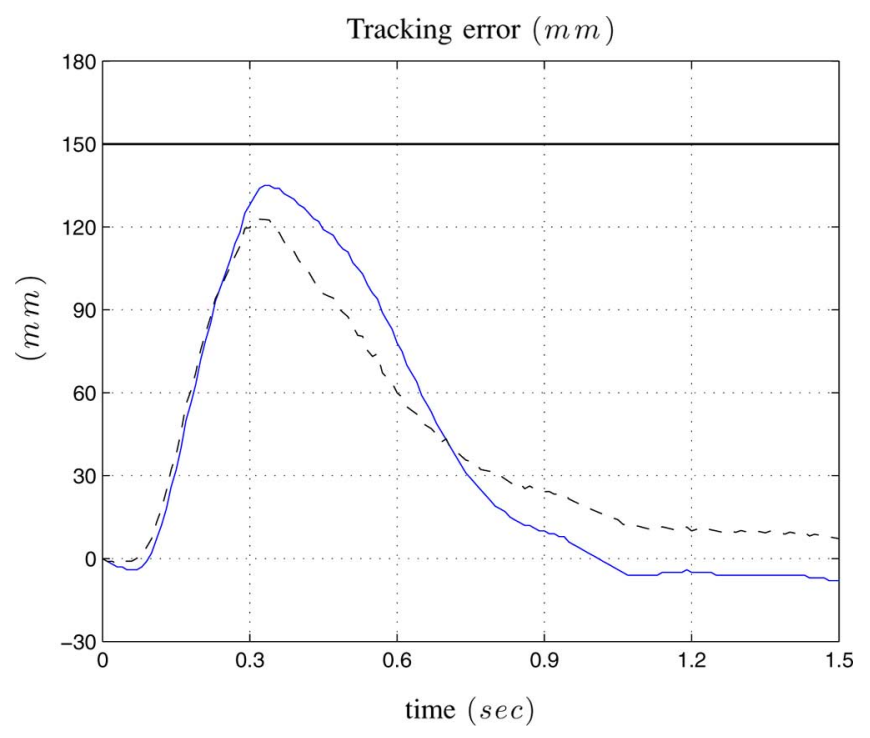

Fig. 15. Tracking error of a real step input: actual (solid line) and predicted by the model (dashed). The HD workspace limit is fixed at $150 \mathrm{~mm}$.

input trajectory. As predicted by the model, even if the maximum error is close to the workspace limitation $\Delta_{\mathrm{WS}}=150 \mathrm{~mm}$, it never reaches such a bound, i.e., during tracking the input the MHI end-effector never reached its workspace boundary.

Finally, to evaluate sinusoidal limitations, experimental trials have been performed using several reference signals $x_{H}(t)=$ $A_{s} \sin (\omega t)$, featuring different amplitudes $A_{s}$ and frequencies $\omega$. In this case, input signals have been generated by a human operator, who, aided by periodic acoustic and visual stimuli, moved the HD's end-effector sideways, approximately describing a time dependent sinusoid. In Fig. 16, the tracking errors of two sinusoidal inputs are shown.

The previous results confirm that the performance indicators introduced in Section V can be useful for evaluating the performance of a real MHI. It was possible to successfully predict 

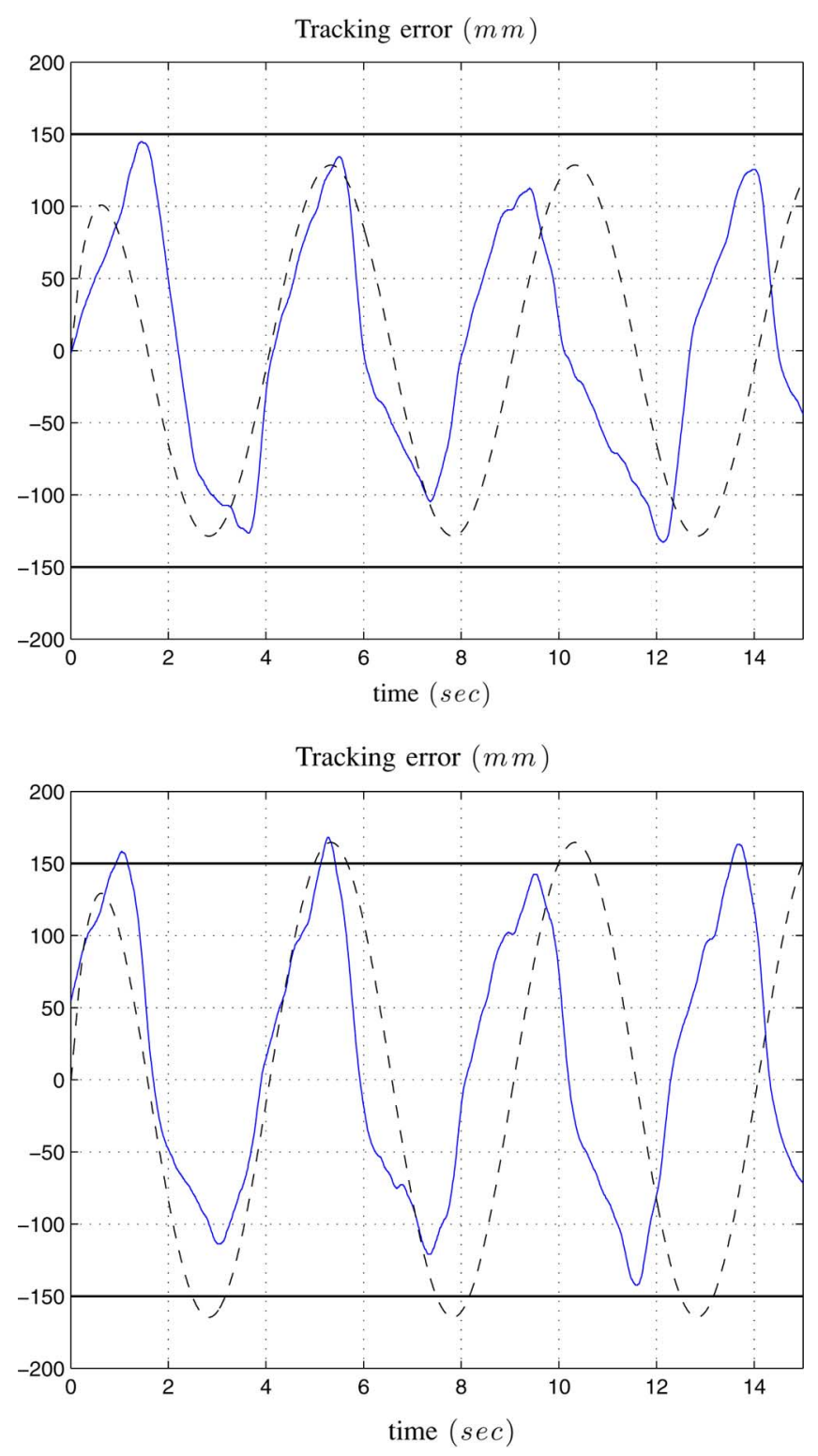

Fig. 16. Actual (solid line) and predicted (dashed line) tracking errors of sinusoidal inputs. On the top $x_{H}(t)=250 \sin (2 \pi 0.2 t)$, on the bottom $x_{H}(t)=300 \sin (2 \pi 0.2 t)$. Thick solid linerepresents the HD workspace limit.

the reaching of the HD workspace limit and a consequent loss of transparency. They also provide a guideline for the controller design, on the basis of a set of performance specifications. It is worth noting that the simple linear model adopted for the theoretical analysis clearly cannot account for a number of issues, which are present in practice (nonlinear dynamics, actuator saturation, communication and processing delays, sensor noise, etc.). Nonetheless, the qualitative behavior predicted by the model is in good agreement with the actual one, and the proposed methodologies of analysis and synthesis can, indeed, be adopted in practice.

\section{Discussion On General InPUT Signals}

So far, the performance analysis has been carried out taking into account only three main classes of input signals. Although

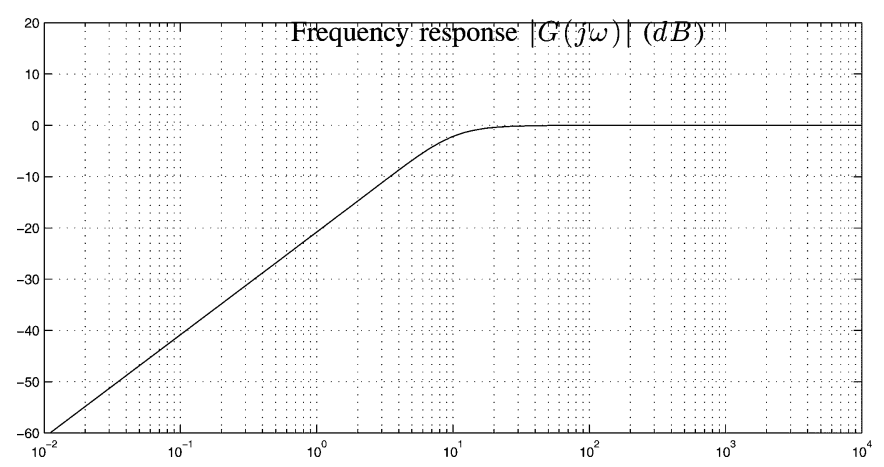

Fig. 17. Frequency response $|G(j \omega)|$ computed for the prototype MHI described in Section VIII.

this may seem to restrict the generality of results, step, ramp, and sinusoidal inputs represent a good benchmark to evaluate the performance of a controlled dynamic system. These results can be generalized to some extent in the frequency domain.

As pointed out in Section V-D, the MP $H(s)$ has a low-pass filter behavior; hence, a necessary condition in order to avoid reaching workspace boundaries is that the frequency content of human hand movements is almost completely contained in the bandwidth of the MP. Experimental measurements revealed that volitional movements involving human hand and elbow typically range from 0 to $10 \mathrm{~Hz}$. Hence, in order for the MHI to track any voluntary operator's movement without rendering spurious forces, the MP should exhibit a bandwidth greater than $10 \mathrm{~Hz}$. On the other hand, a sufficient condition can be found by resorting to the following frequency analysis.

Let $E(\omega)$ and $X_{H}(\omega)$ be the Fourier transform of the tracking error $e(t)$ and $x_{H}(t)$, respectively. By applying the inverse Fourier transform, condition (8) can be rewritten as

$$
|e(t)|=\left|\frac{1}{2 \pi} \int_{-\infty}^{+\infty} E(\omega) e^{j \omega t} d \omega\right|<\Delta_{\mathrm{WS}} .
$$

Since

$$
\left|\frac{1}{2 \pi} \int_{-\infty}^{+\infty} E(\omega) e^{j \omega t} d \omega\right| \leq \frac{1}{2 \pi} \int_{-\infty}^{+\infty}|E(\omega)|\left|e^{j \omega t}\right| d \omega
$$

and being $E(\omega)=G(j \omega) X_{H}(\omega)$ from (9), the following inequality

$$
\int_{0}^{+\infty}\left|G(j \omega) X_{H}(\omega)\right| d \omega<\pi \Delta_{\mathrm{WS}}
$$

is a sufficient condition for (27) to hold. Although this is a conservative constraint, it qualitatively characterizes the maximum admissible generic input $x_{H}(t)$ by setting an upper bound on its frequency content. In fact, the error transfer function $G(s)$ is typically a high-pass filter (see Fig. 17), thus the value of the integral in (28) is finite only if the amplitude of $X_{H}(\omega)$ decreases with the frequency fast enough.

Fig. 18 shows an input trajectory generated by the operator using the prototype MHI (top), and its power spectrum (center). The integral in (28) has been numerically computed yielding the value $464.11 \mathrm{~mm}$, while the upper bound is $\pi \Delta_{\mathrm{WS}}=471.23 \mathrm{~mm}$, being $\Delta_{\mathrm{WS}}=150 \mathrm{~mm}$. Hence, the 

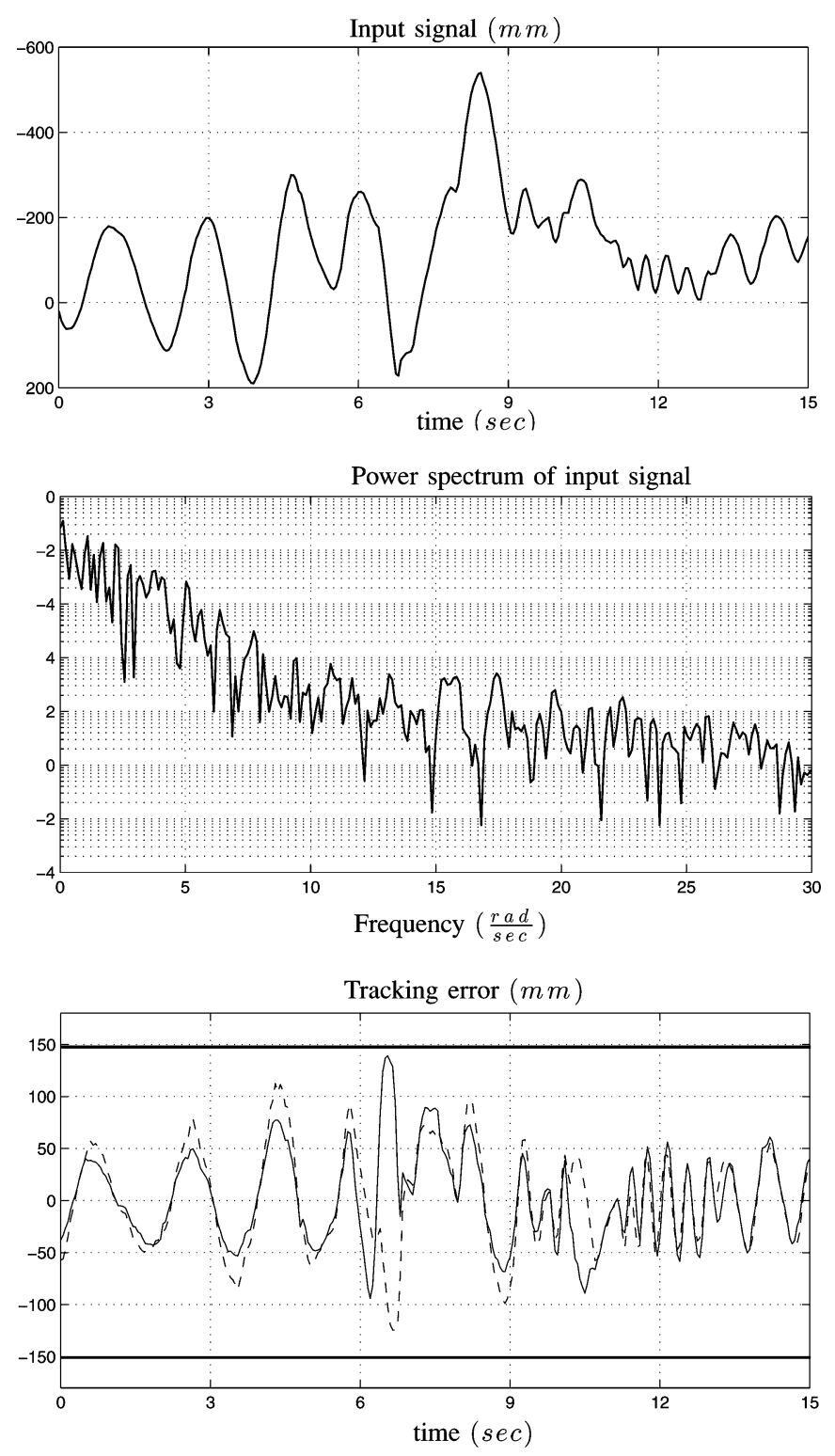

Fig. 18. Top: a generic input trajectory generated by the operator during a VR simulation. Center: the power spectrum of the input trajectory. Bottom: actual (solid line) and predicted (dashed line) tracking error. Thick solid lines represent the workspace limits.

condition (28) holds, and, as shown in the bottom of Fig. 18, the tracking error is confined within workspace boundaries.

As well as for step, ramp, and sinusoidal performance indicators, also condition (28) strictly depends on the model of the MHI. Possible differences between analytic results and real system behaviors can arise accordingly to several factors as pointed out at the end of Section VIII.

\section{CONCLUSION}

Dynamic performance of MHI has been studied along with their limitations and a set of guidelines have been outlined to design MHI controllers. The proposed analysis relates param- eters characterizing an MHI to its performance limitations and presents a procedure on how to design and tune a controller that maximizes the MHI performance from a transparency perspective. In particular, it has been studied what are the controller parameters that allow not to reach the workspace boundaries of the grounded haptic interface while the MPs moves, thus obtaining an unlimited workspace for the whole MHI system. Theoretical results are in good agreement with the real behavior of the MHI prototype used for the experiments, although we used a second-order dynamic model for the MP, which does not take into account many of the nonlinearities present in reality. The proposed results could certainly benefit from more accurate models, but it is our belief that this would also strongly limit the generality of the proposed results, as dynamic models for MPs vary largely on the specific device used.

Work is in progress to extend these results to more complex MHIs as those that involves more than one contact points such as MHIs able to grasp virtual objects and those whose base is a nonholonomic mobile robot.

\section{APPENDIX}

\section{A. Control Design for Ramp Inputs}

Let us consider the transfer function from the end-effector position $x_{H}$ to the tracking error $e$ :

$$
G(s)=\frac{1}{1+H(s) C(s)}=\frac{s(1+\tau s)}{\tau\left(s^{2}+\left[\left(1+K_{v} \tau^{\prime}\right) / \tau\right] s+K_{v} / \tau\right)}
$$

where

$$
K_{v}=\frac{k_{M}}{b_{M}} K_{p}, \quad \tau^{\prime}=\frac{K_{d}}{K_{p}}, \quad \tau=\frac{m_{M}}{b_{M}} .
$$

Let us suppose that the operator moves the end-effector at constant velocity $V_{r}$ :

$$
x_{H}(t)=V_{r} t .
$$

Denoting by $x_{H}(s)$ the Laplace transform of the signal $x_{H}(t)$, the error signal can be computed as

$$
e(s)=G(s) x_{H}(s)=\frac{(1+\tau s) V_{r}}{\tau s\left(s^{2}+\left[\left(1+K_{v} \tau^{\prime}\right) / \tau\right] s+K_{v} / \tau\right)} .
$$

Depending on the values of the controller parameters, the poles of $G(s)$ can be real or complex. Let $\Delta$ be the discriminant

$$
\begin{aligned}
\Delta & =\left(\frac{1+K_{v} \tau^{\prime}}{\tau}\right)^{2}-4 \frac{K_{v}}{\tau} \\
& \triangleq \alpha_{0}+\alpha_{1} K_{d}^{2}+\alpha_{2} K_{d}-4 \alpha_{3} K_{p}
\end{aligned}
$$

where

$$
\alpha_{0}=\frac{1}{\tau^{2}}, \quad \alpha_{1}=\alpha_{3}^{2}, \quad \alpha_{2}=2 \frac{\alpha_{3}}{\tau}, \quad \alpha_{3}=\frac{k_{M}}{m_{M}} .
$$

1) $\Delta<0$ : The system has a pair of complex poles if and only if $\Delta<0$, i.e., if and only if

$$
K_{p}>\frac{\alpha_{3}}{4} K_{d}^{2}+\frac{1}{2 \tau} K_{d}+\frac{1}{4 \alpha_{3} \tau^{2}} .
$$


In this case, the error signal $e(t)$ attains its maximum value during the transient.

2) $\Delta=0$ : If $\Delta=0$, then the system has a pair of coincident real poles at $s=p, p<0$. By antitransforming, the time evolution of the tracking error is

$$
e(t)=A+B t e^{p t}+C e^{p t}, \quad t \geq 0
$$

where

$$
\begin{aligned}
A & =\frac{V_{r}}{K_{v}} \\
B & =\frac{(1+\tau p) V_{r}}{\tau p} \\
C & =-\frac{V_{r}}{\tau p^{2}} .
\end{aligned}
$$

In order to check whether overshoot shows up, let us consider the first-order derivative:

$$
\dot{e}(t)=0 \Longleftrightarrow(1+\tau p)(1+p t)-1=0 .
$$

If

$$
\bar{t} \triangleq-\frac{\tau}{1+\tau p} \geq 0
$$

then, the maximum error is attained at $\bar{t}$. Since

$$
p=-\frac{1}{2}\left(\frac{1}{\tau}+\alpha_{3} K_{d}\right)
$$

in terms of controller parameters, condition (29) becomes $K_{d}>$ $b_{M} / k_{M}$. Hence, if the parameter values are selected so that $\Delta=0$, the choice

$$
K_{d} \leq \frac{b_{M}}{k_{M}}
$$

guarantees that no overshoot shows up.

3) $\Delta>0$ : If $\Delta>0$, the system has real distinct poles: $p_{1}, p_{2} \in(-\infty, 0)$. By antitransforming, the time evolution of the tracking error is

$$
e(t)=A+B e^{p_{1} t}+C e^{p_{2} t}, \quad t \geq 0
$$

where

$$
\begin{aligned}
& A=\frac{V_{r}}{K_{v}} \\
& B=\frac{\left(1+\tau p_{1}\right) V_{r}}{\tau p_{1}\left(p_{1}-p_{2}\right)} \\
& C=\frac{\left(1+\tau p_{2}\right) V_{r}}{\tau p_{2}\left(p_{2}-p_{1}\right)} .
\end{aligned}
$$

Again, let us consider the first-order derivative:

$$
\dot{e}(t)=0 \Longleftrightarrow\left(1+\tau p_{1}\right) e^{p_{1} t}-\left(1+\tau p_{2}\right) e^{p_{2} t}=0 .
$$

a) $\left(1+\tau p_{1}\right)\left(1+\tau p_{2}\right)<0$

If $\left(1+\tau p_{1}\right)$ and $\left(1+\tau p_{2}\right)$ have opposite sign, then $\dot{e}(t) \neq$ 0 for all $t$, and the maximum error is attained at the steady state. b) $\left(1+\tau p_{1}\right)>0$ and $\left(1+\tau p_{2}\right)>0$

Again, if both $p_{1}>-1 / \tau$ and $p_{2}>-1 / \tau$, then $\dot{e}(t) \neq 0$ for all $t$. In fact, without loss of genarality (w.l.o.g.), let us assume

$$
-\frac{1}{\tau}<p_{1}<p_{2}<0
$$

Then

$$
\left(1+\tau p_{1}\right) e^{p_{1} t}<\left(1+\tau p_{2}\right) e^{p_{2} t} \quad \forall t \geq 0 .
$$

c) $\left(1+\tau p_{1}\right)<0$ and $\left(1+\tau p_{2}\right)<0$

Finally, if both $p_{1}<-1 / \tau$ and $p_{2}<-1 / \tau$, then there exists a time instant $\bar{t}$ such that

$$
\left(1+\tau p_{1}\right) e^{p_{1} \bar{t}}=\left(1+\tau p_{2}\right) e^{p_{2} \bar{t}}
$$

and consequently, overshoot shows up. In fact, w.l.o.g., let us assume

$$
p_{1}<p_{2}<-\frac{1}{\tau}<0
$$

then

$$
\lim _{t \rightarrow 0}\left[\left(1+\tau p_{1}\right) e^{p_{1} t}-\left(1+\tau p_{2}\right) e^{p_{2} t}\right]<0 .
$$

But, since $\left(1+\tau p_{1}\right) e^{p_{1} t}$ converges to zero (see later) faster than $\left(1+\tau p_{2}\right) e^{p_{2} t}$, there exists a time $T$ such that

$$
\left(1+\tau p_{1}\right) e^{p_{1} t}-\left(1+\tau p_{2}\right) e^{p_{2} t}>0 \quad \forall t>T .
$$

Hence, by continuity, there exists a time $\bar{t}, 0<\bar{t}<T$ such that $\dot{e}(\bar{t})=0$.

The previous considerations are summarized later. In case of real poles (i.e., when the control parameters $\left(K_{p}, K_{d}\right)$ are such that $\Delta>0$ ), the error signal exhibits overshoot if and only if

$$
p_{1}<-\frac{1}{\tau} \text { and } p_{2}<-\frac{1}{\tau} \text {. }
$$

This condition can be cast in terms of the controller parameters as follows. Since

$$
p_{1,2}=\frac{1}{2}\left(-\frac{1}{\tau}-\alpha_{3} K_{d} \pm \sqrt{\Delta}\right)
$$

inequalities (30) become

$$
\sqrt{\Delta}<\alpha_{3} K_{d}-\frac{1}{\tau} .
$$

Thus, overshoot shows up if and only if inequality (31) holds.

i) First, let us assume

$$
K_{d} \leq \frac{1}{\alpha_{3} \tau}=\frac{b_{M}}{k_{M}} .
$$

Then, since $\Delta>0$, condition (31) is never satisfied. Moreover, in this case, the assumption $\Delta>0$ implies that

$$
K_{p}<\frac{1}{\alpha_{3} \tau^{2}}=\frac{b_{M}^{2}}{k_{M} m_{M}} .
$$

ii) Conversely, let us suppose now that

$$
K_{d}>\frac{1}{\alpha_{3} \tau} .
$$


Then, both sides of inequality (31) are positive, so that the latter can be rewritten as

$$
\Delta<\left(\alpha_{3} K_{d}-\frac{1}{\tau}\right)^{2}
$$

Finally, recalling the expression of $\Delta$, the previous condition becomes

$\alpha_{3}^{2} K_{d}^{2}+2 \frac{\alpha_{3}}{\tau} K_{d}-4 \alpha_{3} K_{p}+\frac{1}{\tau^{2}}<\alpha_{3}^{2} K_{d}^{2}+\frac{1}{\tau^{2}}-2 \frac{\alpha_{3}}{\tau} K_{d}$

that is

$$
K_{p}>\frac{K_{d}}{\tau}
$$

Again, note that the previous inequality is never satisfied for all $K_{p}$ such that

$$
K_{p} \leq \frac{b_{M}^{2}}{k_{M} m_{M}} .
$$

Summarizing, the error signal does not exhibit overshoot if and only if the controller parameters are chosen such that one of the following conditions hold:

$$
\begin{aligned}
& \Delta>0 \text { and } K_{d} \leq \frac{b_{M}}{k_{M}} \\
& \Delta>0 \text { and } K_{p} \leq \frac{b_{M}^{2}}{k_{M} m_{M}} \\
& \Delta>0 \text { and } K_{p} \leq \frac{K_{d}}{\tau} .
\end{aligned}
$$

\section{B. Control Design for Sinusoidal Inputs}

Let us study the dependency of $\|G(j \omega)\|$ on the controller parameters. Recalling the expression of $G(s)$, it follows

$$
G(j \omega)=\frac{-\tau \omega^{2}+j \omega}{-\tau \omega^{2}+K_{v}+j \omega\left(1+K_{v} \tau^{\prime}\right)}
$$

hence

$$
\begin{aligned}
\|G(j \omega)\| & =\frac{\sqrt{\omega^{2}+\tau^{2} \omega^{4}}}{\sqrt{\left(K_{v}-\tau \omega^{2}\right)^{2}+\omega^{2}\left(1+K_{v} \tau^{\prime}\right)^{2}}} \\
& =\frac{\sqrt{\beta_{0}}}{\sqrt{\beta_{0}+\beta_{1} K_{p}^{2}+\beta_{2} K_{d}^{2}-\beta_{3} K_{p}+\beta_{4} K_{d}}}
\end{aligned}
$$

where

$$
\begin{array}{ll}
\beta_{0}=\omega^{2}+\tau^{2} \omega^{4}, & \beta_{1}=\frac{k_{M}^{2}}{b_{M}^{2}}, \quad \beta_{2}=\frac{k_{M}^{2}}{b_{M}^{2}} \omega^{2} \\
\beta_{3}=\frac{2 k_{M} m_{M}}{b_{M}^{2}} \omega^{2}, & \beta_{4}=\frac{2 k_{M}}{b_{M}} \omega^{2} .
\end{array}
$$

Let

$$
K_{p}^{0} \triangleq \frac{\beta_{3}}{2 \beta_{1}}=\frac{m_{M}}{k_{M}} \omega^{2}, \quad K_{d}^{0} \triangleq-\frac{\beta_{4}}{2 \beta_{2}}=-\frac{b_{M}}{k_{M}},
$$

then, the argument of the square root at the denominator can be rewritten as

$$
\mathcal{E}\left(K_{p}, K_{d}\right)=\beta_{1} \beta_{2}\left(\frac{\left(K_{p}-K_{p}^{0}\right)^{2}}{\beta_{2}}+\frac{\left(K_{d}-K_{d}^{0}\right)^{2}}{\beta_{1}}\right) .
$$

Now, it is easily seen that the level curves of $\mathcal{E}\left(K_{p}, K_{d}\right)$ are ellipses in the $\left(K_{p}, K_{d}\right)$ plane:

$$
\mathcal{E}\left(K_{p}, K_{d}\right)=C \Longleftrightarrow \frac{\left(K_{p}-K_{p}^{0}\right)^{2}}{r_{p}^{2}}+\frac{\left(K_{d}-K_{d}^{0}\right)^{2}}{r_{v}^{2}}=1
$$

where $\left(K_{p}^{0}, K_{d}^{0}\right)$ denotes the center and

$$
r_{p}=\sqrt{\frac{C}{\beta_{1}}}, \quad r_{d}=\sqrt{\frac{C}{\beta_{2}}}
$$

represent the radii. Hence, if at a given frequency $\omega$ we desire

$$
\|G(j \omega)\|<v
$$

it is sufficient to choose a pair $\left(K_{p}, K_{d}\right)$, which lies outside the ellipse with center given by (32) and radii given by (33), where

$$
C=\frac{\beta_{0}}{v^{2}} \text {. }
$$

\section{Bounded Acceleration}

Let $W(s)$ be the transfer function from the reference input $x_{H}$ to the MP position $x_{M}$, as defined in (23). In order to assess whether MP acceleration saturates or not, let us study the timeevolution of the acceleration signal $\ddot{x}_{M}(t)$, which, for a ramp input $x_{H}()=V_{r} t$, takes on the form:

$$
\ddot{x}_{M}(t)=\frac{\omega_{n} V_{r}}{\sqrt{1-\zeta^{2}}} e^{-\zeta \omega_{n} t} \sin \left(\omega_{d} t\right)
$$

where $\omega_{d}=\omega_{n} \sqrt{1-\zeta^{2}}$. The maximum value of $\ddot{x}_{M}(t)$ is attained at $t_{\mathrm{MAX}}=\arccos (\zeta) / \omega_{d}$, and

$$
\ddot{x}_{M}\left(t_{\mathrm{MAX}}\right)=\omega_{n} V_{r} e^{-\frac{\zeta}{\sqrt{q-\zeta^{2}}} \arccos (\zeta)} .
$$

The maximum acceleration value $\ddot{x}_{M}\left(t_{\mathrm{MAX}}\right)$ is a decreasing function of the damping ratio $\zeta$, and when $\zeta$ ranges from 0 to $1, \ddot{x}_{M}\left(t_{\mathrm{MAX}}\right)$ ranges from $\omega_{n} V_{r}$ to $\omega_{n} V_{r} / e$. From this analysis, conditions (a) and (b) of Section VII immediately follow. Now, let us suppose that $1<\omega_{n} V_{r} / a_{\operatorname{MAX}}<e$. The value $\ddot{x}_{M}\left(t_{\mathrm{MAX}}\right)$ exceeds the maximum feasible acceleration $a_{\mathrm{MAX}}$ depending on the $\zeta$ value (and consequently, depending on the choice of $K_{p}$ ). Denote by $\phi=\arccos (\zeta)$, (35) can be rewritten as

$$
\ddot{x}_{M}\left(t_{\mathrm{MAX}}\right)=\omega_{n} V_{r} e^{-\frac{\phi}{\tan (\phi)}} .
$$

Clearly, saturation occurs if and only if $\ddot{x}_{M}\left(t_{\mathrm{MAX}}\right) \geq a_{\mathrm{MAX}}$, i.e., if and only if

$$
\phi \leq \log \left(\frac{\omega_{n} V_{r}}{a_{\mathrm{MAX}}}\right) \tan (\phi) .
$$

When $1<\omega_{n} V_{r} / a_{\text {MAX }}<e$, equality (37) admits a unique solution $\phi^{*}$ and inequality (37) is satisfied for each $\phi>\phi^{*}$. Recalling the definition of $\phi$, the previous condition can be recast as $\zeta<\zeta^{*}$, where $\zeta^{*}=\cos \left(\phi^{*}\right)$.

Finally, let us suppose that saturation occurs at a certain time $t_{i}$. This means that $\ddot{x}_{M}\left(t_{i}\right)=a_{\mathrm{MAX}}$. Unfortunately, there is no analytical expression for $t_{i}$. However, it can be bounded as follows. First, notice that clearly $t_{i} \leq t_{\mathrm{MAX}}$. Second, from (34), 
it follows

$$
\begin{aligned}
a_{\mathrm{MAX}} & =\ddot{x}_{M}\left(t_{i}\right)=\frac{\omega_{n} V_{r}}{\sqrt{1-\zeta^{2}}} e^{-\zeta \omega_{n} t_{i}} \sin \left(\omega_{d} t_{i}\right) \\
& \leq \frac{\omega_{n} V_{r}}{\sqrt{1-\zeta^{2}}} \sin \left(\omega_{d} t_{i}\right) .
\end{aligned}
$$

Solving with respect to $t_{i}$, one gets

$$
t_{i} \geq \frac{1}{\omega_{d}} \arcsin \left(\frac{a_{\mathrm{MAX}}}{\omega_{n} V_{r}} \sqrt{1-\zeta^{2}}\right) .
$$

and hence

$$
\arcsin \left(\frac{a_{\mathrm{MAX}}}{\omega_{n} V_{r}} \sqrt{1-\zeta^{2}}\right) \leq \omega_{d} t_{i} \leq \arccos (\zeta) .
$$

For $t \geq t_{i}$, and as long as acceleration saturates, $\ddot{x}_{M}(t)=a_{\mathrm{MAX}}$, hence

$$
x_{M}(t)=x_{M}\left(t_{i}\right)+\dot{x}_{M}\left(t_{i}\right)\left(t-t_{i}\right)+\frac{1}{2} a_{\mathrm{MAX}}\left(t-t_{i}\right)^{2} .
$$

Consequently, the tracking error $e(t)=x_{H}(t)-x_{M}(t)$ evolves as

$$
e(t)=e\left(t_{i}\right)+\dot{e}\left(t_{i}\right)\left(t-t_{i}\right)-\frac{1}{2} a_{\mathrm{MAX}}\left(t-t_{i}\right)^{2}
$$

and its maximum value is

$$
e_{\max }=e\left(t_{i}\right)+\frac{1}{2} \frac{\dot{e}^{2}\left(t_{i}\right)}{a_{\mathrm{MAX}}} .
$$

Since $e\left(t_{i}\right)$ and $\dot{e}\left(t_{i}\right)$ represent the position and velocity error of a linear system, they can be analytically computed as a function of $t_{i}$

$e_{\max }=\frac{2 \zeta}{\omega_{n}} V_{r}+\frac{a_{\mathrm{MAX}}}{\omega_{n}^{2}}\left(\frac{1}{2}\left(\frac{\sqrt{1-\zeta^{2}}}{\tan \left(\omega_{d} t_{i}\right)}-\zeta\right)^{2}-2 \zeta^{2}+1\right)$.

Notice that $e_{\max }$ is a decreasing function of $\omega_{d} t_{i}$; hence, from (38), it follows that (26) is an upper bound for $e_{\max }$.

Finally, in order to have a lower bound on the maximum tracking error in case of acceleration saturation, one may compute $e_{\max }$ in the ideal case of maximum acceleration from initial time, $\ddot{x}_{M}(t)=a_{\mathrm{MAX}}, t \geq 0$. In this case

$$
x_{M}(t)=\frac{1}{2} a_{\mathrm{MAX}} t^{2}
$$

and

$$
e_{\max }=\frac{1}{2} \frac{V_{r}^{2}}{a_{\operatorname{MAX}}}
$$

\section{REFERENCES}

[1] V. Hayward and O. Astley, "Performance measures for haptic interfaces," in Proc. Robot. Res.: 7th Int. Symp., G. Giralt and G. Hirzinger, Eds. New York: Springer-Verlag, 1996, pp. 195-207.

[2] C. Ramstein and V. Hayward, "The pantograph: A large workspace haptic device for a multi-modal human-computer interaction," in Proc. CHI'94, Conf. Human Factors Comput. Syst. ACM/SIGCHI Companion, pp. 5758.

[3] T. Massie and J. Salisbury, "The phantom haptic interface: A device for probing virtual objects," in Proc. ASME Winter Annu. Meet.: Dyn. Syst. Contr., 1994, pp. 295-301.

[4] A. Frisoli, F. Rocchi, S. Marcheschi, A. Dettori, F. Salsedo, and M. Bergamasco, "A new force-feedback arm exoskeleton for haptic interaction in virtual environments," in Proc. World Haptics, Pisa, Italy, Mar. 2005, pp. 195-201.
[5] L. Bouguila, M. Ishii, and M. Sato, "Scaleable SPIDAR: A haptic interface for human-scale virtual environments," in Haptic Human-Computer Interaction, Lecture Notes in Computer Science, vol. 2058, Berlin, Germany: Springer-Verlag/Heidelberg, 2001, pp. 182-193.

[6] M. Ueberle, N. Mock, and M. Buss, "ViSHaRD10, a novel hyperredundant haptic interface," in Proc. 12th Int. Symp. Haptic Interfaces Virtual Environ. Teleoperator Syst. (HAPTICS 2004), Chicago, IL, pp. 5865.

[7] F. Conti and O. Khatib, "Spanning large workspaces using small haptic devices," in Proc. 1st Joint Eurohaptics Conf. Symp. Haptic Interfaces Virtual Environ. Teleoperator Syst., WHC 2005, Pisa, Italy, pp. 183-188.

[8] M. Peshkin, J. E. Colgate, W. Wannasuphoprasit, C. Moore, B. Gillespie, and P. Akella, "Cobot architecture," IEEE Trans. Robot. Autom., vol. 17, no. 4, pp. 377-390, Aug. 2001.

[9] N. Nitzsche, U. D. Hanebeck, and G. Schmidt, "Design issues of mobile haptic interfaces," J. Robot. Syst., vol. 20, no. 9, pp. 549-556, 2003.

[10] F. Barbagli, A. Formaglio, M. Franzini, A. Giannitrapani, and D. Prattichizzo, "An experimental study of the limitations of mobile haptic interfaces," in Experimental Robotics IX: The 9th International Symposyum on Experimental Robotics, (STAR, Springer Tracks in Advanced Robotics Series), O. Khatib and M. Ang, Eds. Berlin Heidelberg: Springer-Verlag, 2006, pp. 533-542.

[11] A. Formaglio, A. Giannitrapani, F. Barbagli, M. Franzini, and D. Prattichizzo, "Performance of mobile haptic interfaces," in Proc. IEEE Conf. Decision Control (IEEE CDC/ECC 2005), Sevilla, Spain, pp. 8343-8348.

[12] D. C. Ruspini, K. Kolarov, and O. Khatib, "The haptic display of complex graphical environments," in Proc. SIGGRAPH97, pp. 345-352.

[13] J. E. Colgate and J. M. Brown, "Factors affecting the $z$-width of a haptic display," in Proc. IEEE Int. Conf. Robot. Autom., San Diego, CA, May1994, pp. 3205-3210.

[14] A. Bicchi and D. Prattichizzo, "Manipulability of cooperating robots with unactuated joints and closed-chain mechanisms," IEEE Trans. Robot. Autom., vol. 16, no. 4, pp. 336-345, Aug. 2000.

[15] T. Yoshikawa, "Manipulability of robotics mechanisms," Int. J. Robot. Res., vol. 4, no. 2, pp. 3-9, 1985.

[16] O. Khatib, "Inertial properties in robotic manipulation: An object-level framework," Int. J. Robot. Res., vol. 14, no. 1, pp. 19-36, 1995.

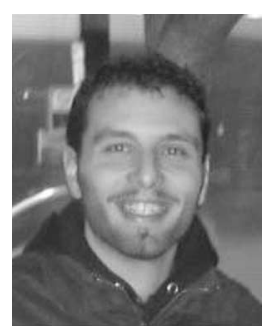

Alessandro Formaglio (A'04) received the M.S. degree in engineering of automation in 2004 and the $\mathrm{Ph} . \mathrm{D}$. degree in robotics and automation in 2008, both from from the University of Siena, Siena, Italy.

During 2004, he was a Research Fellow in the Department of Information Engineering, University of Siena, where he is currently a Research Associate. His current research interests include haptically enabled virtual reality, perception-based haptic rendering and control, virtual object manipulation, and grasping.

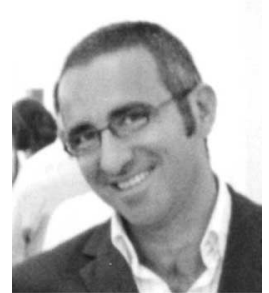

Domenico Prattichizzo (M'93) received the M.S. degree in electronics engineering and the Ph.D. degree in robotics and automation from the University of Pisa, Pisa, Italy, in 1991 and 1995, respectively.

Since 2002, he has been an Associate Professor of Robotics at the University of Siena. During 1994, he was a Visiting Scientist at the Massachusetts Institute of Technology (MIT) Artificial Intelligence Laboratory. He is the author or coauthor of more than 180 papers in the area of robotics and automatic control, and the Co-Editor of two books by STAR, Springer Tracks in Advanced Robotics (Springer, 2003 and 2005). His current research interests include haptics, grasping, visual servoing, and geometric control.

Dr. Prattichizzo was the Co-Chair of the IEEE International Workshop on Control Problems in Robotics and Automation 2002 and of the IEEE International Conference on Robotics and Automation (ICRA) workshop on Multipoint Interaction in Robotics and Virtual Reality 2004. He was the Vice-Chair for Special Issues of the IEEE Technical Committee on Haptics since 2006. Since 2007, he has been an Associate Editor-in-Chief of the IEEE TRANSACTIONS ON HAPTICS. From 2003 to 2007, he was an Associate Editor of the IEEE TRANSACTIONS ON ROBOTICS and IEEE TRANSACTIONS ON CONTROL SYSTEMS TECHNOLOGIES. He was the member of the Editorial Board of many conferences on control and robotics. 


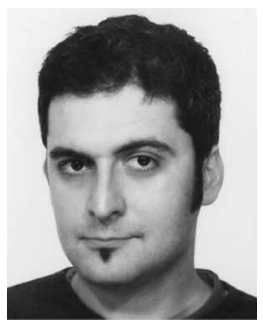

Federico Barbagli received the M.S. degree in computer science from the University of Bologna, Bologna, Italy, in 1998, and the Ph.D. degree in robotics from Scuola Superiore S.Anna, Pisa, Italy, in 2002 .

$\mathrm{He}$ is currently a Senior Engineering Fellow at Hansen Medical, Mountain View, CA, and a Consultant Assistant Professor at the Stanford Computer Science Department. He was a Postdoctoral Fellow at the Stanford Robotics Laboratory from 2002 to 2005, and an Assistant Professor at the University of Siena, Siena, Italy, from 2002 to 2004. His current research interests include haptic interfaces (rendering and controls), medical robotics, and human-machine interaction.

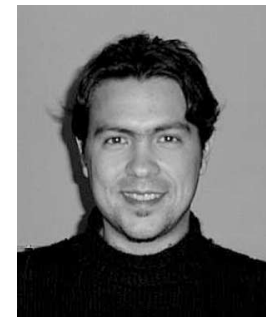

Antonio Giannitrapani (M'07) was born in Salerno, Italy, in 1975. He received the M.S. degree in information engineering in 2000 and the Ph.D. degree in control systems engineering in 2004, both from the University of Siena, Siena, Italy.

Since 2005, he has been with the Department of Information Engineering, University of Siena, where he is currently an Assistant Professor of Robotics. His current research interests include localization and map building for mobile robots, collective motion for teams of autonomous agents, nonlinear estimation techniques for autonomous navigation, and mobile haptic interfaces. 